\title{
Projective Dirac operators, twisted K-theory, and local index formula
}

\author{
Dapeng Zhang*
}

\begin{abstract}
We construct a canonical noncommutative spectral triple for every oriented closed Riemannian manifold, which represents the fundamental class in the twisted K-homology of the manifold. This so-called "projective spectral triple" is Morita equivalent to the well-known commutative spin spectral triple provided that the manifold is spin-c. We give an explicit local formula for the twisted Chern character for K-theories twisted with torsion classes, and with this formula we show that the twisted Chern character of the projective spectral triple is identical to the Poincaré dual of the $\hat{A}$-genus of the manifold.
\end{abstract}

Mathematics Subject Classification (2010). 19K56, 19L50, 58J20.

Keywords. Twisted K-theory, spectral triple, Chern character.

\section{Introduction}

The notion of spectral triple in Connes' noncommutative geometry arises from extracting essential data from the K-homology part of index theory in differential geometry. The following are basic examples of commutative spectral triples:

(i) The spin spectral triple for a $\operatorname{spin}^{\mathrm{c}}$ manifold $M$ with a spinor bundle $S$,

$$
\varsigma_{1}=\left(C^{\infty}(M), \Gamma(S), \not D, \omega\right),
$$

where $\omega$ is the grading operator on $S$.

Throughout this paper, unless otherwise stated explicitly, all vector spaces, algebras, differential forms, and vector bundles except cotangent bundles are considered over the field $\mathbb{C}$ of complex numbers. For example, the notation $C^{\infty}(M)$ is the same as $C^{\infty}(M, \mathbb{C})$. The notation $\Gamma(M, E)$ or $\Gamma(E)$ for a fibre bundle $E$ over $M$ always stands for the space of smooth sections of $E$.

The identity between the analytic and topological indices of $\not D$ is the AtiyahSinger index formula for the $\operatorname{spin}^{\mathrm{c}}$ manifold.

\footnotetext{
*This article is the author's dissertation in publication form. Supported in part by International MaxPlanck Research School (IMPRS).
} 
(ii) The spectral triple for the signature for a Riemannian manifold $M$ :

$$
\varsigma_{2}=\left(C^{\infty}(M), \Omega(M), d+d^{*}, *(-1)^{\frac{\operatorname{deg}(\operatorname{deg}-1)}{2}-\frac{\operatorname{dim} M}{4}}\right) .
$$

For each function $f: \mathbb{N} \rightarrow \mathbb{C}$, we denote by $f(\operatorname{deg}): \Omega(M) \rightarrow \Omega(M)$ the linear operator given by

$$
f(\operatorname{deg}) \omega=f(k) \omega \quad \text { for all } \omega \in \Omega^{k}(M) .
$$

The index formula corresponding to this spectral triple is the Hirzebruch signature formula.

(iii) The spectral triple for the Euler characteristic for a Riemannian manifold $M$ :

$$
\varsigma_{3}=\left(C^{\infty}(M), \Omega(M), d+d^{*},(-1)^{\mathrm{deg}}\right) .
$$

The local index formula corresponding to this spectral triple is the Gauss-BonnetChern theorem.

In fact, every special case of Atiyah-Singer Index theorem corresponds to an instance of commutative spectral triple (with additional structures when necessary). These spectral triples, like $\varsigma_{1}, \varsigma_{2}, \varsigma_{3}$, have many nice properties such as "the five conditions" in Connes [8], and conversely, it is proved that [8] any commutative spectral triple $(\mathcal{A}, \mathscr{H}, D, \gamma)$ satisfying those five conditions is equivalent to a spectral triple consisting of the algebra of smooth functions on a Riemannian manifold $M$, the module of sections of a Clifford bundle over $M$ and a Dirac type operator on it. Furthermore, if $(\mathcal{A}, \mathscr{H}, D, \gamma)$ satisfies an additional important property, the Poincaré duality in $\mathrm{K}$-theory, which means $(\mathcal{A}, \mathcal{H}, D, \gamma)$ represents the fundamental class (i.e., a K-orientation) in $K^{0}(\mathcal{A})$, then it is equivalent to a spin spectral triple $\varsigma_{1}$ for some $\operatorname{spin}^{\mathrm{c}}$ manifold. The spectral triples $\varsigma_{2}$ and $\varsigma_{3}$ do not have the property of Poincaré duality; however, we show in this paper (Corollary 5.4, Theorem 7.1) that for every closed oriented Riemannian manifold there is a canonical noncommutative spectral triple having the property of Poincaré duality in $K^{0}\left(M, W_{3}(M)\right)$, the twisted $\mathrm{K}$ theory of $M$ with local coefficient $W_{3}(M)$, the third integral Stiefel-Whitney class. This canonical spectral triple is called the projective spectral triple on $M$, and its center is unitarily equivalent to $\varsigma_{2}$. The projective spectral triple is Morita equivalent to the spin spectral triple provided that the underlying manifold is $\operatorname{spin}^{\mathrm{c}}$. On the other hand, in the paper of Mathai-Melrose-Singer [20], a so-called projective spin Dirac operator was defined for every Riemannian manifold; however, this operator is in a formal sense. It turns out that the projective spectral triple, in which the Dirac operator is really an operator acting on a Hilbert space, just plays the role of the projective spin Dirac operator.

A spectral triple that gives rise to Poincaré duality in KK-theory first appeared in Kasparov [17]. Kasparov's fundamental class, namely the Dirac element in Definition-Lemma 4.2 in [17], is essentially a spectral triple, although there was no such terminology at that time. The algebra underlying Kasparov's spectral triple 
is noncommutative and $\mathbb{Z}_{2}$-graded, but in many situations things would become much less complicated if the algebra were ungraded, especially when considering its Dixmier-Douady class or passing it to cyclic cohomology class via Connes-Chern character. The projective spectral triple constructed in this paper (Corollary 5.4) has a noncommutative but ungraded algebra, and we show in Section 6 that it is in fact Morita equivalent to that of Kasparov's. To construct such a spectral triple, we first review in Section 3 the definition of spectral triples with certain smoothness property, then introduce in Section 4 the notion of Morita equivalence between them, and then find in Section 5 that local spin spectral triples on small open subsets of a manifold can be glued together, via Morita equivalence, to form a globally defined spectral triple.

The noncommutative algebras underlying projective spectral triples are examples of Azumaya algebras. In Section 1 we review some basic theory on Azumaya algebras, such as the fact that Morita equivalent classes of Azumaya algebras are classified by their Dixmier-Douady classes, and that the K-theory of an Azumaya algebra $\boldsymbol{A}$ coincides with the twisted K-theory of the underlying manifold with the DixmierDouady class of $\boldsymbol{A}$.

Mathai-Stevenson [21] showed that the K-theory (tensoring with $\mathbb{C}$ ) of an Azumaya algebra $\boldsymbol{A}$ is isomorphic to the periodic cyclic homology group of $\boldsymbol{A}$ via ConnesChern character, and that the latter is isomorphic to the twisted de Rham cohomology of the underlying manifold with the Dixmier-Douady class of $\boldsymbol{A}$ via a generalized Connes-Hochschild-Kostant-Rosenberg (CHKR) map.

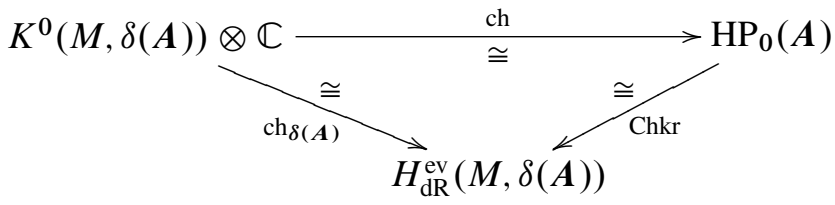

In Section 2, we find an alternative CHKR map (Theorem 2.12) for the special case that the Dixmier-Douady class of $\boldsymbol{A}$ is torsion.

For each algebra $\mathcal{A}$, a finite projective $\mathcal{A}$-module $\mathcal{E}$ as a K-cocycle in the K-theory of $\mathcal{A}$ has a Connes-Chern character $\operatorname{ch}([\mathcal{E}])$ as a cyclic homology class, whereas a spectral triple on $\mathcal{A}$ as a K-cycle in the K-homology group of $\mathcal{A}$ also has a ConnesChern character as a cyclic cohomology class, and the index pairing of a K-cocycle and a K-cycle is identical to the index pairing of their Connes-Chern characters [6], [7]. The main purpose of this paper is to compute the Connes-Chern character of the projective spectral triple and identify it with the Poincaré dual of the $\hat{A}$-genus of the underlying manifold.

In Section 8, with the help of the alternative CHKR map $\rho$ and by applying Poincaré duality, we obtain our main result, a local formula for the Connes-Chern character of the projective spectral triple for every even-dimensional oriented closed Riemannian manifold. 
Acknowledgements. I am very grateful to Bai-Ling Wang. He foresaw the possibility that the projective spin Dirac operator defined by [20] in formal sense can be realized by a certain spectral triple, and introduced his interesting research project to me in 2008. The spectral triple in his mind turned out to be the projective spectral triple constructed in this paper. Without his insight, I would not have been writing this thesis. I would like to thank Adam Rennie for his very helpful remarks about Morita equivalence between various presentations of Kasparov's fundamental class on reading a draft of this paper. I also wish to thank my advisor, Matilde Marcolli, for her many years of encouragement, support, and many helpful suggestions on both this research and other aspects.

\section{Azumaya bundles, twisted K-theory, and twisted cohomology}

Suppose that $X$ is a closed oriented manifold. Let

$$
\mathrm{M}_{n}=\left\{\begin{array}{ll}
\mathrm{M}_{n}(\mathbb{C}), & n=1,2, \ldots, \\
\mathrm{K}(H), & n=\infty,
\end{array} \quad \mathrm{U}_{n}= \begin{cases}\mathrm{U}(n), & n=1,2, \ldots, \\
\mathrm{U}(H), & n=\infty,\end{cases}\right.
$$

where $n$ could be either a positive integer or infinity, $H$ is an infinite-dimensional separable Hilbert space, $\mathrm{K}(H)$ is the $\mathrm{C}^{*}$-algebra of compact operators on $H$, and $\mathrm{U}(H)$ is the topological group of unitary operators with the operator norm topology. Kuiper's theorem states that $\mathrm{U}(H)$ is contractible. Let $\mathrm{PU}_{n}=\mathrm{U}_{n} / \mathrm{U}(1)$ be the projective unitary groups. In particular $\mathrm{PU}(H)=\mathrm{PU}_{\infty}$ is endowed with the topology induced from the norm topology of $\mathrm{U}(H)$.

Let $\operatorname{Aut}\left(\mathrm{M}_{n}\right)$ be the group of automorphisms of the $\mathrm{C}^{*}$-algebra $\mathrm{M}_{n}$.

Fact 1. For every element $g \in \operatorname{Aut}\left(\mathrm{M}_{n}\right)$, there exists $\tilde{g} \in \mathrm{U}_{n}$ such that $g=\operatorname{Ad} \tilde{g}$. For every $u \in \mathrm{U}_{n}, \operatorname{Ad} u=1$ if and only if $u$ is scalar. In other words, as groups $\mathrm{PU}_{n} \cong \operatorname{Aut}\left(\mathrm{M}_{n}\right)$.

Fact 2. If $n$ is finite, $\mathrm{U}_{n} / \mathrm{U}(1) \cong \mathrm{SU}(n) /\left\{z \in \mathbb{C} \mid z^{n}=1\right\}$.

Definition 1.1. An Azumaya bundle over $X$ of rank $n$ (possibly $n=\infty$ ) is a vector bundle over $X$ with fibre $\mathrm{M}_{n}$ and structure group $\mathrm{PU}_{n}$.

Every Azumaya bundle of rank $n$ is associated with a principal $\mathrm{PU}_{n}$-bundle and vice versa.

Definition 1.2. The space $\boldsymbol{A}=\Gamma^{0}(A)$ of continuous sections of an Azumaya bundle $A$ over $X$ forms a $\mathrm{C}^{*}$-algebra called Azumaya algebra over $X$.

The following are examples of Azumaya algebras over $X$ : 
(i) the algebra of complex valued continuous functions $C^{0}(X)$;

(ii) $C^{0}(X) \otimes \mathrm{M}_{n}$;

(iii) if $E$ is a finite rank vector bundle over $X$, the algebra of continuous sections of $\operatorname{End}(E), \Gamma^{0}(\operatorname{End}(E))$;

(iv) if $X$ is an even-dimensional Riemannian manifold, the algebra of continuous sections of the Clifford bundle $\mathrm{Cl}\left(T^{*} X\right), \Gamma^{0}\left(\mathrm{Cl}\left(T^{*} X\right)\right)$;

(v) if $E$ is a real vector bundle over $X$ of even rank with a fiberwise inner product, the algebra of continuous sections of the Clifford bundle $\mathrm{Cl}(E), \Gamma^{0}(\mathrm{Cl}(E))$.

Note that examples (i), (ii) and (iii) are Morita equivalent (in the category of $\mathrm{C}^{*}$ algebras, i.e., strongly Morita equivalent [25], [24]) to $C^{0}(X)$, while example (iv) or (v) is Morita equivalent to $C^{0}(X)$ if and only if $X$ or $E$ is $\operatorname{spin}^{\mathrm{c}}$ respectively.

Fact 3. The center of a finite Azumaya algebra over $X$ is $C^{0}(X)$.

Fact 4. An Azumaya algebra A over $X$ is locally Morita equivalent to $C^{0}(X)$.

The obstruction to an Azumaya algebra being (globally) Morita equivalent to its "center" is characterized by its Dixmier-Douady class:

Definition 1.3. For every Azumaya bundle $\pi: A \rightarrow X$ of rank $n$, there is a cohomology class $\delta(A)$ in $H^{3}(X, \mathbb{Z})$, called the Dixmier-Douady class of $A$, constructed as follows:

Let $\left\{U_{i}\right\}_{i \in I}$ be a good covering of $X$, and write $U_{i_{1} \ldots i_{n}}$ for the intersection of $U_{i_{1}}, U_{i_{2}}, \ldots, U_{i_{n}}$. Suppose that, for all $i \in I$,

$$
\psi_{i}: U_{i} \times \mathrm{M}_{n} \rightarrow \pi^{-1}\left(U_{i}\right)
$$

provide a local trivialization of $A$. Then $\psi_{i}^{-1} \psi_{j}: U_{i j} \times \mathrm{M}_{n} \rightarrow U_{i j} \times \mathrm{M}_{n}$ give rise to the transition functions $g_{i j} \in C^{0}\left(U_{i j}\right.$, Aut $\left.\left(\mathrm{M}_{n}\right)\right)$. Pick $\tilde{g}_{i j} \in C^{0}\left(U_{i j}, \mathrm{U}_{n}\right)$ such that $\operatorname{Ad} \tilde{g}_{i j}=g_{i j}$ and $\tilde{g}_{i j}=\tilde{g}_{j i}^{-1}$. Thus $\operatorname{Ad}\left(\tilde{g}_{i j} \tilde{g}_{j k} \tilde{g}_{k i}\right)=g_{i j} g_{j k} g_{k i}=1$, which implies

$$
\mu_{i j k}:=\tilde{g}_{i j} \tilde{g}_{j k} \tilde{g}_{k i} \in C^{0}\left(U_{i j k}, \mathrm{U}(1)\right) .
$$

Therefore $\mu$ is a Čech 2-cocycle with coefficient sheaf $\mathcal{U}(1): U \mapsto C^{0}(U, \mathrm{U}(1))$, since $(\partial \mu)_{i j k l}=\mu_{j k l} \mu_{i k l}^{-1} \mu_{i j l} \mu_{i j k}^{-1}=1$. The Čech 2-cocycle $\mu$ is also-called the bundle gerbe structure of $A$. The short exact sequence of sheaves

$$
0 \rightarrow \mathbb{Z} \rightarrow \mathcal{R} \stackrel{\exp 2 \pi i \cdot}{\longrightarrow} \mathcal{U}(1) \rightarrow 0,
$$

where $\mathcal{R}$ is the sheaf $U \mapsto C^{0}(U, \mathbb{R})$, induces an isomorphism of Čech cohomology groups

$$
\partial: \breve{H}^{2}(X, \mathcal{U}(1)) \stackrel{\cong}{\longrightarrow} \check{H}^{3}(X, \mathbb{Z}) .
$$


Define the Dixmier-Douady class by $\delta(A):=\partial[\mu]$. More explicitly, pick $v_{i j k} \in$ $C^{0}\left(U_{i j k}, \mathbb{R}\right)$ such that

$$
\exp 2 \pi i v_{i j k}=\mu_{i j k} \text {. }
$$

Then $\exp 2 \pi i(\partial \nu)_{i j k l}=(\partial \mu)_{i j k l}=1$, which implies that $(\partial v)_{i j k l}=v_{j k l}-v_{i k l}+$ $v_{i j l}-v_{i j k} \in \mathbb{Z}$ are locally constant integers on $U_{i j k l}$. Thus, $\delta(A)=[\partial \nu] \epsilon$ $\breve{H}^{3}(X, \mathbb{Z})$.

Definition 1.4. Suppose that $A$ is an Azumaya bundle, that $\boldsymbol{A}$ is the Azumaya algebra corresponding to $A$, and that $P$ is the principal PU-bundle associated to $A$. We say $\delta(\boldsymbol{A})=\delta(P)=\delta(A)$ are the Dixmier-Douady class of $\boldsymbol{A}$ and $P$ respectively.

As a consequence of Kuiper's theorem, we have

Proposition 1.5. For every cohomology class $\delta$ in $H^{3}(X, \mathbb{Z})$, there is a unique (up to isomorphism) infinite rank Azumaya bundle (or algebra) with Dixmier-Douady class $\delta$.

Proposition 1.6. Let $A$ be an Azumaya bundle. If $\delta(A)=0$, then one can choose $\tilde{g}_{i j}$ such that $\tilde{g}_{i j}$ are the transition functions of a certain Hermitian bundle $E$ over $X$, and $A$ is isomorphic to $\mathrm{K}(E)$, the bundle over $X$ with fibres $\mathrm{K}\left(E_{x}\right)$ for all $x \in X$.

Corollary 1.7. An Azumaya algebra A over $X$ is Morita equivalent to $C^{0}(X)$ if and only if $\delta(\boldsymbol{A})=0$.

Corollary 1.8. Two Azumaya algebras $\boldsymbol{A}_{1}$ and $\boldsymbol{A}_{2}$ over $X$ are Morita equivalent if and only if $\delta\left(\boldsymbol{A}_{1}\right)=\delta\left(\boldsymbol{A}_{2}\right)$. Namely, Morita equivalence classes of Azumaya algebras are parameterized by $H^{3}(X, \mathbb{Z})$.

As a consequence of Fact 2, we have

Proposition 1.9. If $A$ is an Azumaya bundle of finite rank $n$, then $n \delta(A)=0$.

For example, suppose that $X$ is a $2 m$-dimensional smooth manifold. The Clifford bundle $\mathrm{Cl}\left(T^{*} X\right)$ is an Azumaya bundle of rank $2^{m}$. Its Dixmier-Douady class $\delta\left(\mathrm{Cl}\left(T^{*} X\right)\right)=W_{3}(X)$ is the third integral Stiefel-Whitney class of $X$, and $2 W_{3}(X)=0$.

Proposition 1.10. If $A_{1}$ and $A_{2}$ are two Azumaya bundles over $X$, then

$$
\delta\left(A_{1} \otimes A_{2}\right)=\delta\left(A_{1}\right)+\delta\left(A_{2}\right) .
$$

Proposition 1.11. If $\boldsymbol{A}$ is an Azumaya algebra, then its opposite algebra $\boldsymbol{A}^{\mathrm{op}}$ is also an Azumaya algebra and

$$
\delta\left(\boldsymbol{A}^{\mathrm{op}}\right)=-\delta(\boldsymbol{A})
$$


Let $\delta$ be a cohomology class in $H^{3}(X, \mathbb{Z})$. Recall that (Rosenberg [26], AtiyahSegal [1]) the twisted $K$-theory $K^{0}(X, \delta)$ can be defined by

$$
K^{0}(X, \delta)=[P \rightarrow \operatorname{Fred}(H)]_{\mathrm{PU}(H)},
$$

the abelian group of homotopy classes of maps $P \rightarrow \operatorname{Fred}(H)$ that are equivariant under the natural action of $\mathrm{PU}(H)$, where $P$ is a principal $\mathrm{PU}(H)$-bundle over $X$ with Dixmier-Douady class $\delta(P)=\delta$; and where Fred $(H)$ is the space of Fredholm operators on $H$. Twisted $\mathrm{K}$-theory can also be defined with $\mathrm{K}$-theory of a $\mathrm{C}^{*}$-algebra:

$$
K^{0}(X, \delta)=K_{0}(\boldsymbol{A}) .
$$

Here $\boldsymbol{A}$ is an (infinite rank) Azumaya algebra over $X$ with Dixmier-Douady class $\delta(\boldsymbol{A})=\delta$. One can also define the twisted $K^{1}$-group by $K^{1}(X, \delta)=K_{1}(\boldsymbol{A})$. The above two definitions of twisted K-theory are equivalent (Rosenberg [26]). We will always use the second definition in this paper.

Proposition 1.12. The direct sum of twisted $K$-groups of $X$,

$$
\bigoplus_{\delta \in H^{3}(X, \mathbb{Z})} K^{\bullet}(X, \delta),
$$

forms a $\mathbb{Z}_{2} \times H^{3}(X, \mathbb{Z})$-bigraded ring. The product $K^{i}\left(X, \delta_{1}\right) \times K^{j}\left(X, \delta_{2}\right) \rightarrow$ $K^{i+j}\left(X, \delta_{1}+\delta_{2}\right)$ is naturally defined.

Definition 1.13. Let $c \in \Omega^{3}(X)$ be a closed 3-form, the twisted de Rham complex is the periodic sequence

$$
\stackrel{d_{c}}{\longrightarrow} \Omega^{\mathrm{ev}}(X) \stackrel{d_{c}}{\longrightarrow} \Omega^{\mathrm{odd}}(X) \stackrel{d_{c}}{\longrightarrow},
$$

where $d_{c} \omega=d \omega+c \wedge \omega$. The twisted de Rham cohomology is $H_{\mathrm{dR}}^{*}(X, c)=$ $H^{*}\left(\Omega^{*}(X), d_{c}\right)$.

Proposition 1.14. If $c$ is a closed 3 -form, then $H_{\mathrm{dR}}^{*}(X, c) \cong H_{\mathrm{dR}}^{*}(X, z c)$ as isomorphic vector spaces for all nonzero $z \in \mathbb{C}$.

In particular, $H_{\mathrm{dR}}^{*}(X, c) \cong H_{\mathrm{dR}}^{*}(X,-c)$ as vector spaces. In fact, in some literatures such as [21], the twisted coboundary $d_{c} \omega$ of $\omega$ is defined by $d \omega-c \wedge \omega$.

Proposition 1.15. If a closed 3-form $c_{1}=c_{2}+d \beta$ for some $\beta \in \Omega^{2}(X)$, then

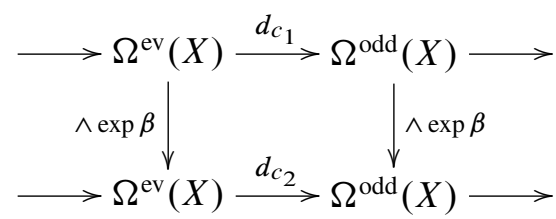

is a chain isomorphism. Therefore $H_{\mathrm{dR}}^{*}\left(X, c_{1}\right) \cong H_{\mathrm{dR}}^{*}\left(X, c_{2}\right)$ as vector spaces. 


\section{Generalized Connes-Hochschild-Kostant-Rosenberg theorem}

In this section, we assume that $M$ is a smooth oriented closed manifold, and that $A$ is an Azumaya bundle over $M$ with a smooth structure in the sense that all the transition functions for the vector bundle $A$ are smooth functions valued in the (Banach) Lie group $\mathrm{PU}_{n}$. Let $\mathcal{A}$ be the space of smooth trace class sections of $A$, then $\mathcal{A}$ is a Fréchet pre-C $\mathrm{C}^{*}$-algebra densely embedded in $\boldsymbol{A}=\Gamma^{0}(A)$. In particular, if the rank $n$ of $A$ is finite, then $\mathcal{A}=\Gamma(A)$.

Given a $\mathrm{PU}_{n}$-connection $\nabla: \Omega^{k}(M, A) \rightarrow \Omega^{k+1}(M, A)$ on $A$, the image of the Dixmier-Douady class $\delta(A)$ in $H_{\mathrm{dR}}^{3}(M, \mathbb{R})$ can be represented by a differential 3-form in terms of the connection and curvature (e.g., Freed [13]) as follows:

Let $\left\{U_{i}\right\}$ be a good open covering of $M$, and $\psi_{i}: U_{i} \times\left.\mathrm{M}_{n} \rightarrow A\right|_{U_{i}}$ be a local trivialization compatible with the smooth structure on $A$. Denote by $g_{j i} \in C^{\infty}\left(U_{i j}, \mathrm{PU}_{n}\right)$ the transition function corresponding to $\psi_{j}^{-1} \psi_{i}$. Pick $\tilde{g}_{j i} \in C^{\infty}\left(U_{i j}, \mathrm{U}_{n}\right)$ such that $\operatorname{Ad} \tilde{g}_{j i}=g_{j i}$ and $\tilde{g}_{j i}=\tilde{g}_{i j}^{-1}$. Let $\theta_{i}$ be the local connection forms of $\nabla$ on $U_{i}$,

$$
\nabla\left(\psi_{i}(O)\right)=\psi_{i}\left(d O+\theta_{i}(O)\right) \text { for all } O \in C^{\infty}\left(U_{i}, \mathrm{M}_{n}\right) .
$$

Then $\theta_{i}=g_{j i}^{-1} \theta_{j} g_{j i}+g_{j i}^{-1} d g_{j i}$. Pick $\tilde{\theta}_{i} \in \Omega^{1}\left(U_{i}, \mathrm{M}_{n}\right)$ if $n \neq \infty$, or pick $\tilde{\theta}_{i} \in$ $\Omega^{1}\left(U_{i}, \mathrm{~B}(H)\right)$ if $n=\infty$ such that $\tilde{\theta}_{i}$ is skew-Hermitian and $\theta_{i}=\operatorname{ad} \tilde{\theta}_{i}$. Thus

$$
\tilde{\theta}_{i}=\tilde{g}_{j i}^{-1} \tilde{\theta}_{j} \tilde{g}_{j i}+\tilde{g}_{j i}^{-1} d \tilde{g}_{j i}+\alpha_{i j},
$$

for some scalar valued 1-form $\alpha_{i j} \in \Omega^{1}\left(U_{i j}\right)$. Let $\omega_{i}$ be the local curvature forms of $\Omega=\nabla^{2}: \Gamma(A) \rightarrow \Omega^{2}(X, A)$ on $U_{i}$,

$$
\Omega\left(\psi_{i}(O)\right)=\psi_{i}\left(\omega_{i}(O)\right) \text { for all } O \in C^{\infty}\left(U_{i}, \mathrm{M}_{n}\right) .
$$

So $\omega_{i}=d \theta_{i}+\theta_{i} \wedge \theta_{i}$ and $\omega_{i}=g_{j i}^{-1} \omega_{j} g_{j i}$. Let $\widetilde{\omega}_{i}=d \tilde{\theta}_{i}+\tilde{\theta}_{i} \wedge \tilde{\theta}_{i}$. Then ad $\widetilde{\omega}_{i}=\omega_{i}$ and $\widetilde{\omega}_{i}=\tilde{g}_{j i}^{-1} \widetilde{\omega}_{j} \tilde{g}_{j i}+d \alpha_{i j}$. Let $\widetilde{\Omega}_{i}=\psi_{i} \widetilde{\omega}_{i} \psi_{i}^{-1}$. Then $\widetilde{\Omega}_{i}=\widetilde{\Omega}_{j}+d \alpha_{i j}$. Since $d \alpha_{i j}+d \alpha_{j k}+d \alpha_{k i}=0, d \alpha$ forms a 2-form valued cocycle, and since the sheaf of 2 -forms is fine (or because of the existence of partition of unity on $M$ ), there exist $\beta_{i} \in \Omega^{2}\left(U_{i}\right)$ such that $2 \pi i\left(\beta_{i}-\beta_{j}\right)=d \alpha_{i j}$. We can define a generalized 2-form by $\widetilde{\Omega}_{i}-2 \pi i \beta_{i}$ on $U_{i}$, and it is globally well defined.

Lemma 2.1. If $A$ is an Azumaya bundle over $M$ with connection $\nabla$ and curvature $\Omega$, then $-\frac{1}{2 \pi i} \nabla\left(\widetilde{\Omega}_{i}-2 \pi i \beta_{i}\right)$ represents the image of the Dixmier-Douady class $\delta(A)$ in $H_{\mathrm{dR}}^{3}(M)$.

Proof. First recall that the Čech-de Rham isomorphism between the third de Rham cohomology $H_{\mathrm{dR}}^{3}(M)$ and Čech cohomology $\breve{H}^{3}(M, \mathbb{C})$ with constant coefficient sheaf $\mathbb{C}$ can be constructed as follows. For any closed 3-form $\tau \in \Omega^{3}(M)$, one can find $\beta(\tau)_{i} \in \Omega^{2}\left(U_{i}\right)$ such that $d \beta(\tau)_{i}=\left.\tau\right|_{U_{i}}$. Since $d \beta(\tau)_{i}-d \beta(\tau)_{j}=$ 0 , one can find $\alpha(\tau)_{i j} \in \Omega^{1}\left(U_{i j}\right)$ such that $\beta(\tau)_{i}-\beta(\tau)_{j}=d \alpha(\tau)_{i j}$. Since 
$d \alpha(\tau)_{i j}+d \alpha(\tau)_{j k}+d \alpha(\tau)_{k i}=0$, one can find $\nu(\tau)_{i j k} \in C^{\infty}\left(U_{i j k}\right)$ such that $(\partial \alpha(\tau))_{i j k}=d \nu(\tau)_{i j k}$ on $U_{i j k}$. Here $\partial$ denotes the coboundary operator on Čech cocycles. Likewise, since $d(\partial \nu(\tau))_{i j k l}=(\partial d \nu(\tau))_{i j k l}=0$, one can find $\delta(\tau)_{i j k l} \in \mathbb{C}$ such that $(\partial v(\tau))_{i j k l}=\delta(\tau)_{i j k l}$. The Čech-de Rham isomorphism $H_{\mathrm{dR}}^{3}(M) \rightarrow$ $\breve{H}^{3}(M, \mathbb{C})$ is given by the correspondence $\tau \mapsto \delta(\tau)$.

Now let $\tau=-\frac{1}{2 \pi i} \nabla\left(\widetilde{\Omega}_{i}-2 \pi i \beta_{i}\right)$. By the Bianchi identity $-\frac{1}{2 \pi i} \nabla\left(\widetilde{\Omega}_{i}-\right.$ $\left.2 \pi i \beta_{i}\right)=d \beta_{i}$. Thus we can choose $\beta(\tau)_{i}=\beta_{i}, \alpha(\tau)_{i j}=\alpha_{i j}, v(\tau)_{i j k}=v_{i j k}$, and $\delta(\tau)_{i j k l}=\delta_{i j k l}$. Therefore it follows that $\tau$ represents the image of $\delta(A)$ in $H_{\mathrm{dR}}^{3}(M)$.

Theorem 2.2. (1) If $A$ is a finite rank Azumaya bundle with connection $\nabla$ and curvature $\Omega$, then there is a unique traceless $\sigma(\Omega) \in \Omega^{2}(M, A)$ such that $\operatorname{ad} \sigma(\Omega)=\Omega$ and $\nabla(\sigma(\Omega))=0$.

(2) Suppose that $A$ is an infinite rank Azumaya bundle associated to a principal $\mathrm{PU}(H)$-bundle $P$ with connection $\nabla$ and curvature $\Omega$, and that $c \in \Omega^{3}(M)$ is a differential form representing the image of the Dixmier-Douady class $\delta(A)$ in $H_{\mathrm{dR}}^{3}(M)$. Then, up to a closed scalar 2-form, there is a unique $\Gamma(P \times \mathrm{PU}(H) \mathrm{B}(H))$-valued 2-form $\sigma(\Omega)$ such that $\operatorname{ad} \sigma(\Omega)=\Omega$ and $-\frac{\nabla(\sigma(\Omega))}{2 \pi i}=c$. Here $\mathrm{PU}(H)$ acts on $\mathrm{B}(H)$ the same way as on $\mathrm{K}(H)$.

Proof. Up to a scalar valued 2-form, $\sigma(\Omega)$ can be defined by $\widetilde{\Omega}_{i}-2 \pi i \beta_{i}$ as in the above lemma. In fact, $\operatorname{ad}\left(\widetilde{\Omega}_{i}-2 \pi i \beta_{i}\right)=\operatorname{ad} \widetilde{\Omega}_{i}=\Omega$, and $-\frac{1}{2 \pi i} \nabla\left(\widetilde{\Omega}_{i}-2 \pi i \beta_{i}\right)$ represents the image of the Dixmier-Douady class $\delta(A)$ in $H_{\mathrm{dR}}^{3}(M)$.

(1) If $A$ is of rank $m<\infty$, we just take $\sigma(\Omega)=\widetilde{\Omega}_{i}-2 \pi i \beta_{i}-\operatorname{tr}\left(\widetilde{\Omega}_{i}-2 \pi i \beta_{i}\right) / m$. The scalar 3-form $\nabla(\sigma(\Omega))$ must be 0 since it is traceless. The uniqueness of $\sigma(\Omega)$ is obvious.

(2) Suppose that $A$ is of infinite rank. There exists a scalar 2-form $\eta$ such that

$$
-\frac{1}{2 \pi i} \nabla\left(\widetilde{\Omega}_{i}-2 \pi i \beta_{i}\right)-c=d \eta .
$$

We can take $\sigma(\Omega)=\widetilde{\Omega}_{i}-2 \pi i \beta_{i}-2 \pi i \eta$.

Recall that if $\mathscr{B}$ is a pre- $\mathrm{C}^{*}$-algebra densely embedded in a $\mathrm{C}^{*}$-algebra $\boldsymbol{B}$, then $K_{0}(\mathscr{B})=K_{0}^{\mathrm{alg}}(\mathscr{B})$ is naturally isomorphic to $K_{0}(\boldsymbol{B})$. If $\mathscr{B}$ is a unital Fréchet algebra, we define $K_{1}(\mathscr{B})$ to be the abelian group of the equivalence classes of $\mathrm{GL}_{\infty}(\mathscr{B})$. We say that $u, v \in \mathrm{GL}_{\infty}(\mathscr{B})$ are equivalent if there is a piecewise $C^{1}$-path in $\mathrm{GL}_{\infty}(\mathscr{B})$ joining $u$ and $v$. The definition of $K_{1}(\mathscr{B})$ can be extended to the case of non-unital algebras so that the six-term exact sequence property holds. For Azumaya algebras, $K_{*}(\mathcal{A})$ is naturally isomorphic to $K_{*}(\boldsymbol{A})=K^{*}(M, \delta(\boldsymbol{A}))$. We refer to [6], [7], [18] for the definitions of Hochschild, cyclic and periodic cyclic homologies and cohomologies. We denote the Hochschild boundary map and the Connes boundary by $b$ and $\mathrm{B}$, respectively. 
Definition 2.3. Following Gorokhovsky [15], define two maps

Chkr: $\bigoplus_{k \text { even }} \bar{C}_{k}^{\text {red }}(\mathcal{A}) \rightarrow \Omega^{\mathrm{ev}}(M) \quad$ and $\quad$ Chkr $: \bigoplus_{k \text { odd }} \bar{C}_{k}^{\text {red }}(\mathcal{A}) \rightarrow \Omega^{\text {odd }}(M)$

by the JLO-type ([16]) formula

$$
\operatorname{Chkr}\left(a_{0}, a_{1}, \ldots, a_{k}\right)=\int_{s \in \Delta^{k}} \operatorname{tr}\left(a_{0} e^{-s_{0} \sigma(\Omega)}\left(\nabla a_{1}\right) e^{-s_{1} \sigma(\Omega)} \ldots\left(\nabla a_{k}\right) e^{-s_{k} \sigma(\Omega)}\right) d s .
$$

Here $\bar{C}_{0}^{\text {red }}(\mathcal{A})=\mathcal{A}$ and $\bar{C}_{j}^{\text {red }}(\mathcal{A})=\mathcal{A}^{+} \hat{\otimes} \mathcal{A}^{\hat{\otimes} j}$ for all $j \neq 0$, with $\mathcal{A}^{+}$being the unitalization of $\mathcal{A}$ and $\hat{\otimes}$ the projective tensor product of locally convex topological algebras. Here and subsequently, if $V=\bigoplus_{i} V^{i}$ is a $\mathbb{Z}$-graded vector space, we write $V^{\mathrm{ev}}=\bigoplus_{k} V^{2 k}$ and $V^{\text {odd }}=\bigoplus_{k} V^{2 k+1}$.

The generalized CHKR theorem of Mathai-Stevenson is as follows.

Proposition 2.4 (Mathai-Stevenson [21]). (1) The map Chkr in (1) induces a quasiisomorphism between the two complexes

$$
\stackrel{b}{\rightarrow} \bar{C}_{\mathrm{ev}}^{\mathrm{red}}(\mathcal{A}) \stackrel{b}{\rightarrow} \bar{C}_{\mathrm{odd}}^{\mathrm{red}}(\mathcal{A}) \stackrel{b}{\rightarrow} \text { and } \stackrel{0}{\rightarrow} \Omega^{\mathrm{ev}}(M) \stackrel{0}{\rightarrow} \Omega^{\text {odd }}(M) \stackrel{0}{\rightarrow},
$$

and hence isomorphisms $\mathrm{HH}_{\mathrm{ev}}(\mathcal{A}) \cong \Omega^{\mathrm{ev}}(M)$ and $\mathrm{HH}_{\mathrm{odd}}(\mathcal{A}) \cong \Omega^{\mathrm{odd}}(M)$.

(2) The map Chkr induces a quasi-isomorphism between the complex

$$
\stackrel{\mathrm{b}+\mathrm{B}}{\longrightarrow} \bar{C}_{\mathrm{ev}}^{\mathrm{red}}(\mathcal{A}) \stackrel{\mathrm{b}+\mathrm{B}}{\longrightarrow} \bar{C}_{\mathrm{odd}}^{\mathrm{red}}(\mathcal{A}) \stackrel{\mathrm{b}+\mathrm{B}}{\longrightarrow} \text { or equivalently } \stackrel{\mathrm{B}}{\rightarrow} \mathrm{HH}_{\mathrm{ev}}(\mathcal{A}) \stackrel{\mathrm{B}}{\rightarrow} \mathrm{HH}_{\mathrm{odd}}(\mathcal{A}) \stackrel{\text { B }}{\rightarrow}
$$

and the twisted de Rham complex

$$
\stackrel{d_{c}}{\longrightarrow} \Omega^{\mathrm{ev}}(M) \stackrel{d_{c}}{\longrightarrow} \Omega^{\mathrm{odd}}(M) \stackrel{d_{c}}{\longrightarrow},
$$

and hence an isomorphism

$$
\text { Chkr: HP.(A) } \stackrel{(}{\longrightarrow} H_{\mathrm{dR}}^{\bullet}(M, c) \quad(\bullet=\mathrm{ev}, \text { odd }),
$$

where $c=-\frac{\nabla(\sigma(\Omega))}{2 \pi i}$ is a representative of the image of $\delta(A)$ in $H_{d R}^{3}(M)$.

(3) The Connes-Chern character $\mathrm{ch}: K_{\bullet}(\mathcal{A}) \otimes \mathbb{C} \rightarrow \mathrm{HP}_{\bullet}(\mathcal{A})$ and the twisted Chern character

$$
\operatorname{ch}_{\delta(A)}=\operatorname{Chkr} \circ \operatorname{ch}: K^{\bullet}(M, \delta(A)) \otimes \mathbb{C} \rightarrow H_{\mathrm{dR}}^{\bullet}(M, c)
$$

are isomorphisms.

If $\delta(\mathcal{A})$ is a torsion class, then there is an alternative formula for the map Chkr, which we will see is closely related to the relative Chern character ([3]) of Clifford 
modules. From now on we take $c=0$; then by Theorem 2.2, up to a closed 2-form, there is a unique $\sigma(\Omega)$ such that ad $\sigma(\Omega)=\Omega$ and $\nabla(\sigma(\Omega))=0$.

Define $\psi_{k}: \mathcal{A}^{\hat{\otimes} k} \rightarrow \mathcal{A} \hat{\otimes}_{C^{\infty}(M)} \Omega^{k}(M)$ by letting

$$
\begin{gathered}
\psi_{-1}=0, \quad \psi_{0}=1, \quad \psi_{1}\left(a_{1}\right)=\nabla a_{1}, \quad \psi_{2}\left(a_{1}, a_{2}\right)=\left(\nabla a_{1}\right)\left(\nabla a_{2}\right)+a_{1} \sigma(\Omega) a_{2}, \\
\psi_{k}\left(a_{1}, \ldots, a_{k}\right)=\left(\nabla a_{1}\right) \psi_{k-1}\left(a_{2}, \ldots, a_{k}\right)+a_{1} \sigma(\Omega) a_{2} \psi_{k-2}\left(a_{3}, \ldots, a_{k}\right)
\end{gathered}
$$

for all $k \geq 2$. In other words, $\psi_{k}\left(a_{1}, \ldots, a_{k}\right)$ is obtained as follows: Consider all partitions $\pi$ of the ordered set $\left\{a_{1}, \ldots, a_{k}\right\}$ into blocks, where each block contains either one or two elements. Assign to each block $\left\{a_{i}\right\}$ of $\pi$ a term of the form $\nabla a_{i}$, and to each block $\left\{a_{j}, a_{j+1}\right\}$ of $\pi$ a term of the form $a_{j} \sigma(\Omega) a_{j+1}$. Then let $\psi_{k, \pi}$ be the product of these terms and $\psi_{k}\left(a_{1}, \ldots, a_{k}\right)$ be the sum of $\psi_{k, \pi}$ over all such partitions. So in its expansion, $\psi_{k}\left(a_{1}, \ldots, a_{k}\right)$ consists of a Fibonacci number of summands. Then define $\rho_{k}: \mathcal{A}^{\hat{\otimes} k+1} \rightarrow \Omega^{k}(M)$ by

$$
\rho_{k}\left(a_{0}, \ldots, a_{k}\right)=\frac{1}{k !} \operatorname{tr}\left(a_{0} \psi_{k}\left(a_{1}, \ldots, a_{k}\right)\right)
$$

for all $k \geq 0$. Note that $\rho$ depends on the choice of connection $\nabla$ and, for infinite rank Azumaya bundles, the choice of $\sigma(\Omega)$, so we will write the complete form $\rho_{\sigma}^{\nabla}$ for $\rho$ when we need to specify $\nabla$ and $\sigma$. We have the following results about $\rho$.

Lemma 2.5. $\rho \circ b=0$.

Lemma 2.6. If $\delta(\mathcal{A})$ is a torsion class, then

$$
(-1)^{k-1} \rho_{k}\left(a_{0}, \ldots, a_{k}\right)+\rho_{k}\left(a_{k}, a_{0}, \ldots, a_{k-1}\right)=d \operatorname{tr}\left(a_{0} \psi_{k-1}\left(a_{1}, \ldots a_{k-1}\right) a_{k}\right)
$$

for all $k \geq 0$ and $a_{i} \in \mathcal{A}$.

Proof. Noticing that $d \circ \operatorname{tr}=\operatorname{tr} \circ \nabla$, it suffices to show

$$
(-1)^{k-1} a_{0} \psi_{k}\left(a_{1}, \ldots, a_{k}\right)+\psi_{k}\left(a_{0}, \ldots, a_{k-1}\right) a_{k}=\nabla\left(a_{0} \psi_{k-1}\left(a_{1}, \ldots, a_{k-1}\right) a_{k}\right)
$$

for all $a_{i} \in \mathcal{A}^{+}$. In fact, it is easy to see that (4) is true for $k=0,1,2$. Suppose that (4) holds for all $k \leq m$ for some $m$. Then using $\nabla^{2}=\operatorname{ad} \sigma(\Omega)$ and the Bianchi identity $\nabla(\sigma(\Omega))=0$, we have

$$
\begin{aligned}
\nabla\left(a_{0} \psi_{m}\right. & \left.\left(a_{1}, \ldots, a_{m}\right) a_{m+1}\right) \\
= & \nabla a_{0} \psi_{m}\left(a_{1}, \ldots, a_{m}\right) a_{m+1}+a_{0} \nabla \psi_{m}\left(a_{1}, \ldots, a_{m}\right) a_{m+1} \\
& +(-1)^{m} a_{0} \psi_{m}\left(a_{1}, \ldots, a_{m}\right) \nabla a_{m+1} \\
= & \nabla a_{0} \psi_{m}\left(a_{1}, \ldots, a_{m}\right) a_{m+1}+a_{0} \nabla\left(\nabla a_{1} \psi_{m-1}\left(a_{2}, \ldots, a_{m}\right)\right) a_{m+1} \\
& +a_{0} \nabla\left(a_{1} \sigma(\Omega) a_{2} \psi_{m-2}\left(a_{3}, \ldots, a_{m}\right)\right) a_{m+1} \\
& +(-1)^{m} a_{0} \psi_{m}\left(a_{1}, \ldots, a_{m}\right) \nabla a_{m+1} \\
= & \psi_{m+1}\left(a_{0}, \ldots, a_{m}\right) a_{m+1}-a_{0} a_{1} \sigma(\Omega) \psi_{m-1}\left(a_{2}, \ldots, a_{m}\right) a_{m+1} \\
& -a_{0} \nabla a_{1} \nabla \psi_{m-1}\left(a_{2}, \ldots, a_{m}\right) a_{m+1}
\end{aligned}
$$




$$
\begin{aligned}
& +a_{0} \nabla a_{1} \sigma(\Omega) a_{2} \psi_{m-2}\left(a_{3}, \ldots, a_{m}\right) a_{m+1} \\
& +a_{0} a_{1} \sigma(\Omega) \nabla a_{2} \psi_{m-2}\left(a_{3}, \ldots, a_{m}\right) a_{m+1} \\
& +a_{0} a_{1} \sigma(\Omega) a_{2} \nabla \psi_{m-2}\left(a_{3}, \ldots, a_{m}\right) a_{m+1} \\
& +(-1)^{m} a_{0} \psi_{m}\left(a_{1}, \ldots, a_{m}\right) \nabla a_{m+1} \\
& =\psi_{m+1}\left(a_{0}, \ldots, a_{m}\right) a_{m+1}-a_{0} a_{1} \sigma(\Omega) \psi_{m-1}\left(a_{2}, \ldots, a_{m}\right) a_{m+1} \\
& +(-1)^{m} a_{0} \nabla a_{1} \psi_{m}\left(a_{2}, \ldots, a_{m}, 1\right) a_{m+1} \\
& -a_{0} \nabla a_{1} \psi_{m}\left(1, a_{2}, \ldots, a_{m}\right) a_{m+1} \\
& +a_{0} \nabla a_{1} \sigma(\Omega) a_{2} \psi_{m-2}\left(a_{3}, \ldots, a_{m}\right) a_{m+1} \\
& +a_{0} a_{1} \sigma(\Omega) \nabla a_{2} \psi_{m-2}\left(a_{3}, \ldots, a_{m}\right) a_{m+1} \\
& +(-1)^{m} a_{0} a_{1} \sigma(\Omega) a_{2} \psi_{m-1}\left(a_{3}, \ldots, a_{m}, 1\right) a_{m+1} \\
& +a_{0} a_{1} \sigma(\Omega) a_{2} \psi_{m-1}\left(1, a_{3}, \ldots, a_{m}\right) a_{m+1} \\
& +(-1)^{m} a_{0} \psi_{m}\left(a_{1}, \ldots, a_{m}\right) \nabla a_{m+1} \\
& =\psi_{m+1}\left(a_{0}, \ldots, a_{m}\right) a_{m+1}-a_{0} a_{1} \sigma(\Omega) \psi_{m-1}\left(a_{2}, \ldots, a_{m}\right) a_{m+1} \\
& +(-1)^{m} a_{0} \nabla a_{1} \psi_{m-2}\left(a_{2}, \ldots, a_{m-1}\right) a_{m} \sigma(\Omega) a_{m+1} \\
& +a_{0} a_{1} \sigma(\Omega) \nabla a_{2} \psi_{m-2}\left(a_{3}, \ldots, a_{m}\right) a_{m+1} \\
& +(-1)^{m} a_{0} a_{1} \sigma(\Omega) a_{2} \psi_{m-3}\left(a_{3}, \ldots, a_{m-1}\right) a_{m} \sigma(\Omega) a_{m+1} \\
& +a_{0} a_{1} \sigma(\Omega) a_{2} \sigma(\Omega) a_{3} \psi_{m-3}\left(a_{4}, \ldots, a_{m}\right) a_{m+1} \\
& +(-1)^{m} a_{0} \psi_{m}\left(a_{1}, \ldots, a_{m}\right) \nabla a_{m+1} \\
& =\psi_{m+1}\left(a_{0}, \ldots, a_{m}\right) a_{m+1} \\
& +(-1)^{m} a_{0} \nabla a_{1} \psi_{m-2}\left(a_{2}, \ldots, a_{m-1}\right) a_{m} \sigma(\Omega) a_{m+1} \\
& +(-1)^{m} a_{0} a_{1} \sigma(\Omega) a_{2} \psi_{m-3}\left(a_{3}, \ldots, a_{m-1}\right) a_{m} \sigma(\Omega) a_{m+1} \\
& +(-1)^{m} a_{0} \psi_{m}\left(a_{1}, \ldots, a_{m}\right) \nabla a_{m+1} \\
& =\psi_{m+1}\left(a_{0}, \ldots, a_{m}\right) a_{m+1}+(-1)^{m} a_{0} \psi_{m+1}\left(a_{1}, \ldots, a_{m+1}\right) \text {. }
\end{aligned}
$$

Thus identity (4) is proved by induction.

Lemma 2.7. If $\mathcal{A}$ is unital, then $\rho_{k+1} \circ \mathrm{B}_{k}=d \circ \rho_{k}$ on $\mathcal{A}^{\hat{\otimes} k+1}$.

Proof. Recall that

$$
\mathrm{B}=(1-\lambda) \circ s \circ N,
$$

where $\lambda$ is the cyclic permutation

$$
\lambda\left(a_{0}, \ldots, a_{n}\right)=(-1)^{n}\left(a_{n}, a_{0}, \ldots, a_{n-1}\right),
$$

$N$ is the sum of all cyclic permutations

$$
N_{k}=1+\lambda+\cdots+\lambda^{k}
$$


and $s_{k}: C_{k}(\mathcal{A}) \rightarrow C_{k+1}(\mathcal{A})$ is the extra degeneracy operator

$$
s\left(a_{0}, \ldots, a_{k}\right)=\left(1, a_{0}, \ldots, a_{k}\right) .
$$

Thus we have

$$
\begin{aligned}
\rho \circ \mathrm{B}\left(a_{0}, \ldots, a_{k}\right) & =\rho \circ(1-\lambda) \circ \sum_{i=0}^{k}(-1)^{k i}\left(1, a_{i}, \ldots, a_{i-1}\right) \\
& =\frac{1}{(k+1) !} \sum_{i=0}^{k}(-1)^{k i}(-1)^{k} d \operatorname{tr}\left(\psi\left(a_{i}, \ldots, a_{i-2}\right) a_{i-1}\right) \\
& =\frac{1}{(k+1)} \sum_{i=0}^{k}(-1)^{k(i-1)} d \rho\left(a_{i-1}, \ldots, a_{i-2}\right) \\
& =d \circ \rho\left(a_{0}, \ldots, a_{k}\right),
\end{aligned}
$$

where the second and the last equality follow from Lemma 2.6.

Corollary 2.8. $\rho_{k+1} \circ \mathrm{B}_{k}=d \circ \rho_{k}$ on $\bar{C}_{k}^{\text {red }}(\mathcal{A})$.

We now take a look at what happens for formula (3) when we choose different connections and different $\sigma$ 's. Let $\nabla_{t}=\nabla_{0}+t \theta$ be a family of $\mathrm{PU}_{n}$-connections on the Azumaya bundle $A$, where $t \in I=[0,1]$ and $\theta$ is a generalized 1-form. Denote by $\Omega_{t}$ the curvature of $\nabla_{t}$. If $A$ has finite rank, then the choice of $\sigma$ is unique and $\sigma\left(\Omega_{t}\right)$ is a smooth family of traceless generalized 2-forms. If $A$ has infinite rank, then given $\sigma_{0}\left(\Omega_{0}\right)$ and $\sigma_{1}\left(\Omega_{1}\right)$ with $\nabla_{i}\left(\sigma_{i}\left(\Omega_{i}\right)\right)=0$ and ad $\sigma_{i}\left(\Omega_{i}\right)=\Omega_{i}$, where $i=0,1$, we can always find a smooth family $\sigma_{t}\left(\Omega_{t}\right)$ with $\nabla_{t}\left(\sigma_{t}\left(\Omega_{t}\right)\right)=0$ and ad $\sigma_{t}\left(\Omega_{t}\right)=\Omega_{t}$. In fact, consider the projection $\tilde{\pi}: M \times I \rightarrow I$ and the pull-back bundle $\tilde{\pi}^{*} A=A \times I$ over $M \times I$ with connection $\tilde{\nabla}=\nabla_{t}+d t \partial_{t}$. The curvature on $\tilde{\pi}^{*} A$ is $\widetilde{\Omega}=\Omega_{t}+\theta d t$. Since $\tilde{\pi}^{*} A$ is also an Azumaya bundle with torsion Dixmier-Douady class, we can choose some $\sigma(\widetilde{\Omega})$ with $\widetilde{\nabla}(\sigma(\widetilde{\Omega}))=0$ and $\operatorname{ad} \sigma(\widetilde{\Omega})=\widetilde{\Omega}$. Suppose that $\sigma(\widetilde{\Omega})=\Lambda_{t}+\eta_{t} d t$, where $\Lambda_{t}$ and $\eta_{t}$ are a smooth family of generalized 2 -forms and a smooth family of generalized 1-forms on $M$. Then ad $\Lambda_{t}=\Omega_{t}$, ad $\eta_{t}=\theta$ and $\nabla_{t} \Lambda_{t}=0$. Therefore, by Theorem 2.2, $\sigma_{0}\left(\Omega_{0}\right)-\Lambda_{0}$ and $\sigma_{1}\left(\Omega_{1}\right)-\Lambda_{1}$ are closed scalar 2 -forms. Then we can take a smooth family of generalized 2 -forms

$$
\sigma_{t}\left(\Omega_{t}\right)=\Lambda_{t}+(1-t)\left(\sigma_{0}\left(\Omega_{0}\right)-\Lambda_{0}\right)+t\left(\sigma_{1}\left(\Omega_{1}\right)-\Lambda_{1}\right) .
$$

We have $\nabla_{t}\left(\sigma_{t}\left(\Omega_{t}\right)\right)=0$ and ad $\sigma_{t}\left(\Omega_{t}\right)=\Omega_{t}$.

Consider the good open covering $\left\{U_{i}\right\}$ of $M$ again. Suppose that

$$
\left(\sigma_{0}\left(\Omega_{0}\right)-\Lambda_{0}\right)-\left(\sigma_{1}\left(\Omega_{1}\right)-\Lambda_{1}\right)=d \eta_{i}
$$


on $U_{i}$ for some scalar 1-form $\eta_{i}$ and let

$$
\tilde{\sigma}(\tilde{\Omega})=\sigma_{t}\left(\Omega_{t}\right)+\eta_{t} d t+\eta_{i} d t .
$$

Then we have $\tilde{\nabla}(\tilde{\sigma}(\tilde{\Omega}))=0$ and $\operatorname{ad} \tilde{\sigma}(\tilde{\Omega})=\widetilde{\Omega}$.

Following Mathai-Stevenson [21], let

$$
K\left(a_{0}, \ldots, a_{k}\right)=\int_{I} d t \wedge\left(\iota_{t} \rho_{\tilde{\sigma}}^{\tilde{\nabla}}\left(a_{0}, \ldots, a_{k}\right)\right)
$$

on $U_{i}$. Here the functions $a_{0}, \ldots, a_{k}$ inside $\rho_{\tilde{\sigma}}^{\tilde{\nabla}}()$ are considered as functions on $U_{i} \times I$ which are constant in the $t$ direction. We have the following lemma.

Lemma 2.9. The map $K$ defined above is a chain homotopy. We have the formula

$$
\rho_{\sigma_{1}}^{\nabla_{1}}-\rho_{\sigma_{0}}^{\nabla_{0}}=K \circ(b+\mathrm{B})-d \circ K
$$

on $\bar{C}_{*}^{\text {red }}\left(\mathcal{A} \mid U_{i}\right)$.

Proof. By Lemma 2.5 and Corollary 2.8,

$$
\begin{aligned}
K \circ(b+\mathrm{в})-d \circ K & =K \circ \mathrm{\textrm {B }}-d \circ K \\
& =\int_{I} d t \wedge \iota_{\partial_{t}} \circ d \circ \rho_{\tilde{\sigma}}^{\tilde{\nabla}}-d \int_{I} d t \wedge \iota_{\partial_{t}} \circ \rho_{\tilde{\sigma}}^{\tilde{\nabla}} \\
& =\int_{I} d t \wedge\left(\iota_{\partial_{t}} \circ d+d \circ \iota_{\partial_{t}}\right) \circ \rho_{\tilde{\sigma}}^{\tilde{\nabla}} \\
& =\int_{I} d t \wedge \frac{\partial}{\partial t} \circ \rho_{\tilde{\sigma}}^{\tilde{\nabla}} \\
& =\rho_{\sigma_{1}}^{\nabla_{1}}-\rho_{\sigma_{0}}^{\nabla_{0}} .
\end{aligned}
$$

Theorem 2.10. If $\delta(\mathcal{A})$ is a torsion class, then the map $\rho_{k}$ in (3) induces a quasiisomorphism between the complexes

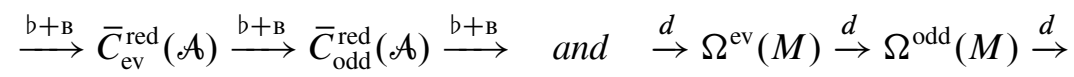

and its induced isomorphism

$$
\rho: \mathrm{HP} .(\mathcal{A}) \stackrel{\cong}{\longrightarrow} H_{\mathrm{dR}}^{\bullet}(M)
$$

coincides with the map Chkr in (2).

Proof. First, by Lemma 2.5 and Corollary 2.8, we see that $\rho$ is a chain map. Then the homotopy formula in Lemma 2.9 and Mathai-Stevenson's spectral sequence argument, [21], on the Čech-de Rham bicomplex prove the theorem. 
Because the map $\rho$ is degree-preserving, we have

Corollary 2.11. If $\delta(\mathcal{A})$ is a torsion class, then the map $\rho$ in (3) induces a chain isomorphism

$$
\left(\mathrm{HH}_{*}(\mathcal{A}), \mathrm{\text {в}}\right) \rightarrow\left(\Omega^{*}(M), d\right) .
$$

Theorem 2.12. If $\delta(\mathcal{A})$ is a torsion class, then the map $\rho$ in (3) induces a homomorphism

$$
\rho: C_{*}^{\lambda}(\mathcal{A}) \rightarrow \Omega^{*}(M) / d\left(\Omega^{*-1}(M)\right),
$$

where $C_{*}^{\lambda}(\mathcal{A})$ is the Connes complex of $\mathcal{A}$ (cf. [6], [7]) and an isomorphism

$$
\rho: \mathrm{HP}_{\bullet}(\mathcal{A}) \stackrel{\cong}{\longrightarrow} H_{\mathrm{dR}}^{\bullet}(M) \text {, }
$$

which coincides with Chkr in (2).

Proof. First, by Lemma 2.6 we see that the induced homomorphism

$$
C_{*}^{\lambda}(\mathcal{A}) \rightarrow \Omega^{*}(M) / d\left(\Omega^{*-1}(M)\right)
$$

is well defined.

Next we show that the induced map $\rho:$ HP. $(\mathcal{A}) \rightarrow H_{\mathrm{dR}}^{\bullet}(M)$ is well defined. Note that $\rho_{k} \circ b=0$ on $C_{k+1}(\mathcal{A})$. This means that the map $C_{*}^{\lambda}(\mathcal{A}) / b\left(C_{*+1}^{\lambda}(\mathcal{A})\right) \rightarrow$ $\Omega^{*}(M) / d\left(\Omega^{*-1}(M)\right)$ is well defined. Then it suffices to show that the images of $\mathrm{HP}_{*}(\mathcal{A})$ under the map $\rho$ are represented by closed forms. We prove this only for the case of even degree, the odd degree case is similar. Since ch: $K_{0}(\mathscr{A}) \rightarrow \mathrm{HP}_{0}(\mathscr{A})$ is an isomorphism, elements of $\mathrm{HP}_{0}(\mathcal{A})$ are generated by $\operatorname{ch}[p]$ for $[p] \in K_{0}(\mathcal{A})$ (here we may suppose that $\mathcal{A}$ is unital for simplicity). The periodic Connes-Chern character $\operatorname{ch}[p]$ is represented by a sequence of cyclic cycles $\left\{\operatorname{ch}_{0}^{\lambda}(p), \operatorname{ch}_{2}^{\lambda}(p), \ldots\right\}$, where

$$
\operatorname{ch}_{2 m}^{\lambda}(p)=(-1)^{m} \frac{(2 m) !}{m !} \operatorname{tr}\left(p^{\otimes 2 m+1}\right) \in C_{2 m}^{\lambda}(\mathcal{A}) .
$$

Observe that $p(\nabla p)^{2 i+1} p=0$ for all idempotent $p$ and $i \geq 0$. Then

$$
\rho_{2 k}\left(\operatorname{ch}_{2 k}^{\lambda}(p)\right)=(-1)^{k} \frac{(2 k) !}{k !} \operatorname{tr}\left(p \psi_{2 k}(p, \ldots, p)\right)=(-1)^{k} \frac{(2 k) !}{k !} \operatorname{tr}\left(p \psi_{2}(p, p)^{k}\right)
$$

because in the expansion of $p \psi_{2 k}(p, \ldots, p) p$ any term that has a factor $p(\nabla p)^{2 i+1} p$ vanishes. Since $\nabla\left(p \psi_{2}(p, p)\right)=0$, it follows that $\rho(\operatorname{ch}[p])$ is a closed form for all $[p] \in K_{0}(\mathcal{A})$.

Finally, since the periodic cyclic homology of $\mathcal{A}$ defined from the $C^{\lambda}$-complex is the same as that defined from the (b, B)-bicomplex, by Theorem 2.10 , the induced homomorphism $\rho$ : HP.(A) $\rightarrow H_{\mathrm{dR}}^{\bullet}(M)$ is an isomorphism identical to Chkr in (2). 


\section{Spectral analysis of spectral triples}

In this section we review the definition and some analytical properties of spectral triples. Note that a slight modification to the standard definition of spectral triple (cf. [10]) is made so that it will be more convenient to develop the theory in this paper. In fact, in definition 3.6 we require that the second entry $\mathscr{H}$ of a spectral triple $(\mathcal{A}, \mathscr{H}, D)$ to be an $\mathscr{A}$-module as well as the smooth Sobolev domain of $D$, instead of the Hilbert space $\overline{\mathscr{H}}$ the norm completion of $\mathscr{H}$. So in application in differential geometry, spectral triples defined this way operate directly with smooth sections of vector bundles. For a spectral triple in the conventional sense, that would be a strong requirement, as strong as the smoothness condition in Appendix B in [11].

Suppose that $D$ is a densely defined self-adjoint operator on a Hilbert space $H$ and that $D$ has compact resolvent. Let $\mu_{1}>\mu_{2}>\cdots$ be the list of eigenvalues of $\left(D^{2}+1\right)^{-1}$ in decreasing order, and $V_{i} \subset H$ be the eigenspace corresponding to $\mu_{i}$ for each $i$. Then every vector $v \in H$ can be uniquely represented as a sequence $\left(v_{1}, v_{2}, \ldots\right)$ with $v_{i} \in V_{i}$ and $\sum_{i}\left\|v_{i}\right\|^{2}<\infty$, and vice versa.

For every $s \geq 0$, consider the following subspaces of $H$,

$$
W^{s}(D)=\left\{\left(v_{1}, v_{2}, \ldots\right) \in H \mid \sum_{i} \mu_{i}^{-s}\left\|v_{i}\right\|^{2}<\infty\right\},
$$

with the norm $\left\|\left(v_{1}, v_{2}, \ldots\right)\right\|_{s}=\sqrt{\sum_{i} \mu_{i}^{-s}\left\|v_{i}\right\|^{2}}$;

$$
W^{s, p}(D)=\left\{\left(v_{1}, v_{2}, \ldots\right) \in H \mid \sum_{i} \mu_{i}^{-s p / 2}\left\|v_{i}\right\|^{p}<\infty\right\} \text { for all } p>0,
$$

with the norm $\left\|\left(v_{1}, v_{2}, \ldots\right)\right\|_{s, p}=\left(\sum_{i} \mu_{i}^{-s p / 2}\left\|v_{i}\right\|^{p}\right)^{1 / p}$; and

$$
W^{s, \infty}(D)=\left\{\left(v_{1}, v_{2}, \ldots\right) \in H \mid \sup _{i} \mu_{i}^{-s / 2}\left\|v_{i}\right\|<\infty\right\},
$$

with the norm $\left\|\left(v_{1}, v_{2}, \ldots\right)\right\|_{s, \infty}=\sup _{i} \mu_{i}^{-s / 2}\left\|v_{i}\right\| . W^{s}=W^{s, 2}$ has a natural Hilbert space structure.

Proposition 3.1 (Rellich). For each $\varepsilon>0$, the inclusion $W^{s+\varepsilon} \rightarrow W^{s}$ is compact.

Proposition 3.2. $W^{1} \subset H$ is the domain of the self-adjoint operator $D$ and $D: W^{1} \rightarrow H$ is a Fredholm operator.

Let $W^{\infty}=\bigcap_{s>0} W^{s}$, then $W^{\infty}$ is a Fréchet space with a family of norms $\|\cdot\|_{s}$. It is easy to see that restricted to $W^{\infty}$ the mapping $D: W^{\infty} \rightarrow W^{\infty}$ is continuous with respect to the Fréchet space topology.

We say the operator $D$ is finitely summable or has spectral dimension less than $2 d$ (for some real number $d>0$ ) if $\left(D^{2}+1\right)^{-d}$ is a trace class operator.

Theorem 3.3. Suppose that $D$ has finite spectral dimension. If $T \in B(H)$ is a bounded operator that maps $W^{\infty}$ into $W^{\infty}$, then the restricted mapping $T: W^{\infty} \rightarrow$ $W^{\infty}$ is also continuous. 
This theorem can be proved by the following lemmas.

Lemma 3.4 (Sobolev embeddings). If D has spectral dimension less than $2 d$, then we have the obvious estimate

$$
\|v\|_{s, \infty} \leq\|v\|_{s, p} \leq\left(\sum_{j} \mu_{j}^{d}\right)^{1 / p}\|v\|_{s+\frac{2 d}{p}, \infty} \text { for all } v \in H, s \geq 0, p>0,
$$

i.e., there are bounded embeddings $W^{s+\frac{2 d}{p}, \infty} \subset W^{s, p} \subset W^{s, \infty}$.

Lemma 3.5. Suppose that $D$ has finite spectral dimension.

(1) Let $T: W^{\infty} \rightarrow W^{\infty}$ be a continuous operator. Suppose that for each $j$,

$$
T\left(0, \ldots, 0, v_{j}, 0, \ldots\right)=\left(t_{1 j} v_{j}, t_{2 j} v_{j}, \ldots\right) \text { for all } v_{j} \in V_{j}
$$

where $\left(t_{i j}\right)$ is an infinite matrix with entries $t_{i j} \in \operatorname{Hom}\left(V_{j}, V_{i}\right)$. Then $\left(t_{i j}\right)$ satisfies the property: for any $s>0$ there exist $C$ and $r>0$ such that

$$
\left\|\sum_{i} \mu_{i}^{-s} t_{i j}\right\|<C+\mu_{j}^{-r} \text { for all } j
$$

(2) Conversely any matrix $\left(t_{i j}\right)$ with entries $t_{i j} \in \operatorname{Hom}\left(V_{j}, V_{i}\right)$ satisfying the above property represents a continuous operator $T: W^{\infty} \rightarrow W^{\infty}$.

Proof. (1) For any $v \in W^{\infty}$, we see that $\sum_{j=0}^{n} v_{j} \rightarrow v$ in $W^{s}$ as $n \rightarrow \infty$. Therefore $\sum_{j=0}^{n} v_{j} \rightarrow v$ in $W^{\infty}$. Because $T$ is continuous, it follows that $T\left(\sum_{j=0}^{n} v_{j}\right)=$ $\left(\sum_{j=0}^{n} t_{1 j} v_{j}, \sum_{j=0}^{n} t_{2 j} v_{j}, \ldots\right) \rightarrow T v$, hence $(T v)_{i}=\sum_{j} t_{i j} v_{j}$. Suppose the claim is not true. Then there must exist $s>0$ such that for any $C$ and $n$ there is $j(n, C)$ satisfying

$$
\left\|\sum_{i} \mu_{i}^{-s} t_{i j(n, C)}\right\|>C+\mu_{j(n, C)}^{-n} .
$$

Thus one may find an increasing sequence $\{j(n)\}$ such that

$$
\left\|\sum_{i} \mu_{i}^{-s} t_{i j(n)}\right\|>\mu_{j(n)}^{-n}
$$

For each $j$, pick $u_{j} \in V_{j}$ such that $\left\|u_{j(n)}\right\|=\left\|\sum_{i} \mu_{i}^{-s} t_{i j(n)}\right\|^{-1}<\mu_{j(n)}^{n}$ and $\left\|\sum_{i} \mu_{i}^{-s} t_{i j(n)} u_{j(n)}\right\|=1$, while $u_{j}=0$ if $j \neq j(n)$ for all $n$. Then, because of the finite spectral dimension, $u=\sum_{j} u_{j} \in W^{\infty}$, but $T\left(\sum_{j=0}^{n} u_{j}\right)$ does not converge in $W^{2 s}$ as $n \rightarrow \infty$, which yields a contradiction.

(2) Suppose the matrix $\left(t_{i j}\right)$ has that property. Define $T: W^{\infty} \rightarrow W^{\infty}$ by

$$
(T v)_{i}=\sum_{j} t_{i j} v_{j} \quad \text { for all } v \in W^{\infty} \text {. }
$$


For any sequence $u(n) \in W^{\infty}$, we now prove that if $u(n) \rightarrow 0$ as $n \rightarrow \infty$, then $T u(n) \rightarrow 0$. For any $s>0$,

$$
\begin{aligned}
\|T u(n)\|_{2 s} & =\left\|\sum_{i} \mu_{i}^{-s} \sum_{j} t_{i j} u_{j}(n)\right\| \\
& \leq \sum_{j}\left\|\sum_{i} \mu_{i}^{-s} t_{i j} u_{j}(n)\right\| \\
& \leq \sum_{j}\left(C+\mu_{j}^{-r}\right)\left\|u_{j}(n)\right\|=C\|u(n)\|_{0,1}+\|u(n)\|_{r, 1} .
\end{aligned}
$$

Since $u(n) \rightarrow 0$ in $W^{\infty}$ implies $\|u(n)\|_{s^{\prime}} \rightarrow 0$ for all $s^{\prime}$, using Lemma 3.4 it follows that $\|T u(n)\|_{2 s} \rightarrow 0$ for all $s$. So this implies $T u(n) \rightarrow 0$ and so $T: W^{\infty} \rightarrow W^{\infty}$ is a continuous operator.

For any pre-Hilbert space $\mathscr{H}$, we define the $*$-algebra $B(\mathscr{H})=\{T \in B(\overline{\mathscr{H}}) \mid$ $\left.T(\mathscr{H}) \subset \mathscr{H}, T^{*}(\mathscr{H}) \subset \mathscr{H}\right\}$.

Definition 3.6. A triple $(\mathcal{A}, \mathscr{H}, D)$ is said to be a unital spectral triple if it is given by a unital pre-C $C^{*}$-algebra $\mathscr{A}$, a pre-Hilbert space $\mathscr{H}$ with a norm-continuous unital $*$ representation $\mathcal{A} \rightarrow B(\mathscr{H})$, and a self-adjoint operator $D$ on $\overline{\mathscr{H}}$ called Dirac operator with the following properties:

(i) $D$ has compact resolvent.

(ii) $W^{\infty}(D)=\mathscr{H}$.

(iii) Under the representation of $\mathcal{A}$, the commutator $[D, a]: \mathscr{H} \rightarrow \mathscr{H}$ is normbounded for each $a \in \mathcal{A}$.

Besides, we always assume that $\mathcal{A}$ is a locally convex topological $*$-algebra with a topology finer than the norm topology of $\mathcal{A}$, and the representation $\mathcal{A} \times \mathscr{H} \rightarrow \mathcal{H}$ is jointly continuous with respect to the locally convex topology of $\mathcal{A}$ and the Fréchet topology of $\mathscr{H}$.

Note that if $(\mathcal{A}, \mathcal{H}, D)$ is a unital spectral triple, then $W^{1}(D)$, the domain of $D$, also forms a left $\mathcal{A}$-module because of the last condition in the definition.

A spectral triple $(\mathscr{A}, \mathcal{H}, D)$ is said to be even if there is a $\mathbb{Z}_{2}$-grading on $\mathscr{H}$,

$$
\mathscr{H}=\mathscr{H}_{+} \oplus \mathscr{H}_{-},
$$

such that the grading operator

$$
\gamma=\left[\begin{array}{cc}
\operatorname{id}_{\mathscr{H}_{+}} & 0 \\
0 & -\mathrm{id}_{\mathscr{H}_{-}}
\end{array}\right]: \mathscr{H} \rightarrow \mathscr{H}
$$

commutes with all $a \in \mathcal{A}$ and anti-commutes with $D$. Spectral triples equipped with no such gradings are said to be $o d d$.

Two spectral triples $\left(\mathcal{A}_{1}, \mathscr{H}_{1}, D_{1}\right)$ and $\left(\mathscr{A}_{2}, \mathscr{H}_{2}, D_{2}\right)$ with isomorphic algebras $\mathcal{A}_{1} \cong \mathcal{A}_{2}$ are said to be unitarily equivalent if there is a unitary operator 
$U: \overline{\mathscr{H}}_{1} \rightarrow \overline{\mathscr{H}}_{2}$ intertwining the two representations of $\mathcal{A}_{i}$ and the two Dirac operators $D_{i}$ in an obvious way. For the even case the unitary operator $U$ also needs to be grade preserving.

\section{Morita equivalence of spectral triples}

In this section we introduce the notion of Morita equivalence of spectral triples. Let $\mathcal{A}$ be a pre- $\mathrm{C}^{*}$-algebra. Recall that a right $\mathcal{A}$-module $S$ is called a pre-Hilbert (or Hermitian) right $\mathcal{A}$-module if there is an $\mathcal{A}$-valued inner product $(\cdot, \cdot): \mathcal{S} \times S \rightarrow \mathcal{A}$ such that for all $x, y \in \mathcal{S}, a \in \mathcal{A}$,

(i) $(x, y)=(y, x)^{*}$,

(ii) $(x, y a)=(x, y) a$,

(iii) $(x, x) \geq 0$, and $(x, x)=0$ only if $x=0$.

The norm on $\mathcal{S}$ is given by $\|x\|=\|(x, x)\|^{\frac{1}{2}}$, and its norm completion $\overline{\mathcal{S}}$ (which is automatically a pre-Hilbert right $\overline{\mathcal{A}}$-module) is called a Hilbert right $\overline{\mathcal{A}}$-module. Hilbert left modules can be defined in the same manner. In particular, every Hilbert space is a Hilbert $\mathbb{C}$-module.

If $S$ is a pre-Hilbert right $\mathcal{A}$-module, then its conjugate space

$$
\varsigma^{*}=\left\{f_{x}:=(x, \cdot) \mid x \in \delta\right\}
$$

is a pre-Hilbert left $\mathcal{A}$-module, and for all $x, y \in \delta, a \in \mathcal{A}$,

$$
a f_{x}:=a(x, \cdot)=f_{x\left(a^{*}\right)}, \quad\left(f_{x}, f_{y}\right):=(y, x), \quad\left(f_{x}, a f_{y}\right)=a\left(f_{x}, f_{y}\right) .
$$

If $S$ is a pre-Hilbert right $\mathcal{A}$-module, $B_{\mathcal{A}}(\overline{\mathcal{S}})$ denotes the $*$-algebra of all module homomorphisms $T: \overline{\mathcal{S}} \rightarrow \overline{\mathcal{S}}$ for which there is an adjoint module homomorphism $T^{*}: \bar{\delta} \rightarrow \bar{\delta}$ with $(T x, y)=\left(x, T^{*} y\right)$ for all $x, y \in \bar{S}$. Define

$$
B_{\mathcal{A}}(S)=\left\{T \in B_{\mathscr{A}}(\bar{S}) \mid T(S) \subset S, T^{*}(S) \subset S\right\} .
$$

In particular, if $\mathcal{A}=\mathbb{C}$, then $B_{\mathbb{C}}(\mathcal{S})=B(\mathcal{S})$. If $\mathcal{A}$ is unital and $S$ is unital and finitely generated, then $B_{\mathcal{A}}(S)$ in fact consists of all $\mathcal{A}$-endomorphisms of $S$, i.e., $B_{\mathscr{A}}(\mathcal{S})=\operatorname{End}_{\mathcal{A}}(\mathcal{S})$.

Suppose that $\mathcal{A}$ and $\mathcal{B}$ are unital pre- $\mathrm{C}^{*}$-algebras. Let $\mathcal{E}$ be a unital finitely generated projective right $\mathcal{A}$-module. Then as a summand of a free module, one can endow $\mathscr{E}$ with a pre-Hilbert module structure (which is unique up to unitary $\mathcal{A}$-isomorphism). Suppose that $\mathscr{B}$ acts on $\mathscr{E}$ on the left, and the representation $\mathscr{B} \rightarrow B_{\mathcal{A}}(\mathscr{E})$ is unital, $*$-preserving and norm-continuous. We also assume that $\mathscr{E}$ is endowed with the topology induced from the locally convex topology of $\mathcal{A}$, that $\mathscr{B}$ is a locally convex topological $*$-algebra with a topology finer than the norm topology, and that the representation $\mathscr{B} \times \mathscr{E} \rightarrow \mathcal{E}$ is jointly continuous with respect to the locally convex 
topologies on $\mathscr{B}$ and $\mathcal{E}$. We call such a $\mathscr{B}$-A-bimodule with the above structure a finite Kasparov $\mathscr{B}$-A-module and introduce the following definition.

Definition 4.1. Suppose that $\sigma=(\mathcal{A}, \mathcal{H}, D)$ is a unital spectral triple. A $\sigma$ connection on a finite Kasparov $\mathscr{B}$-AA-module $\mathcal{E}$ is a linear mapping

$$
\nabla: \mathcal{E} \rightarrow \mathcal{E} \otimes_{\mathcal{A}} B(\mathscr{H})
$$

with the following properties:

(i) $\nabla(\xi a)=(\nabla \xi) a+\xi \otimes_{\mathcal{A}}[D, a]$,

(ii) $(\xi, \nabla \varepsilon)-(\nabla \xi, \varepsilon)=[D,(\xi, \varepsilon)]$

for all $a \in \mathcal{A}$, and $\xi, \varepsilon \in \mathcal{E}$.

Note that by the notation $(\nabla \xi, \varepsilon)$, which is a bit ambiguous, we actually mean $(\varepsilon, \nabla \xi)^{*} \in B(\mathscr{H})$.

Since $\mathcal{E}$ is finitely generated, it follows that for each $b \in \mathscr{B}$, the commutator $[\nabla, b]$ corresponds to an element in $B\left(\mathscr{E} \otimes_{\mathcal{A}} \mathscr{H}\right)$. It also follows that any two $\sigma$-connections on $\mathscr{E}$ differ by a Hermitian element in $B\left(\mathscr{E} \otimes_{\mathcal{A}} \mathscr{H}\right)$.

From the above data $(\mathcal{A}, \mathscr{H}, D, \mathscr{B}, \mathcal{E}, \nabla)$, one can construct a new spectral triple $\sigma^{\mathcal{E}}=\left(\mathscr{B}, \mathscr{H}^{\mathcal{E}}, D^{\mathcal{E}}\right)$ for $\mathscr{B}$ (cf. Connes [7], §VI.3). The pre-Hilbert space $\mathscr{H}^{\mathcal{E}}$ is $\mathscr{E} \otimes_{\mathcal{A}} \mathcal{H}$ with inner product given by

$$
\left\langle\xi_{1} \otimes_{\mathcal{A}} h_{1}, \xi_{2} \otimes_{\mathcal{A}} h_{2}\right\rangle=\left\langle h_{1},\left(\xi_{1}, \xi_{2}\right) h_{2}\right\rangle \quad \text { for all } \xi_{i} \in \mathcal{E}, h_{i} \in \mathcal{H} .
$$

The Dirac operator $D^{\mathscr{E}}$ on $\overline{\mathscr{H}^{\mathcal{E}}}$ is given by

$$
D^{\mathcal{E}}\left(\xi \otimes_{\mathcal{A}} h\right)=\xi \otimes_{\mathcal{A}} D h+(\nabla \xi) h \quad \text { for all } \xi \in \mathcal{E}, h \in W^{1}(D) .
$$

It is easy to see that the commutator $\left[D^{\mathcal{E}}, b\right]=[\nabla, b] \in B\left(\mathcal{E} \otimes_{\mathcal{A}} \mathscr{H}\right)$ is bounded. To verify that $D^{\mathcal{E}}$ is self-adjoint with domain $\mathcal{E} \otimes_{\mathcal{A}} W^{1}(D)$, that $W^{\infty}\left(D^{\mathcal{E}}\right)=\mathscr{H}^{\mathcal{E}}$, and that $D^{\mathscr{E}}$ has compact resolvent, it is adequate to check choosing one particular $\sigma$-connection on $\mathcal{E}$, because bounded perturbations do not affect these conclusions.

Recall that a universal connection on a pre-Hilbert right $\mathcal{A}$-module $S$ is a linear mapping $\nabla: S \rightarrow S \otimes_{\mathcal{A}} \Omega_{u}^{1}(\mathcal{A})$ that satisfies the Leibniz rule

$$
\nabla(s a)=(\nabla s) a+\delta_{u} a,
$$

and $\nabla$ is said to be Hermitian if

$$
(s, \nabla \varepsilon)-(\nabla s, \varepsilon)=\delta_{u}(s, \varepsilon) .
$$

Here $\Omega_{u}^{1}(\mathcal{A})$ is the space of universal 1-forms of $\mathcal{A}$, and involutions $\left(\delta_{u} a\right)^{*}$ are set to be $-\delta_{u}\left(a^{*}\right)$. Cuntz and Quillen [12] showed that only projective modules admit universal connections. Given a universal Hermitian connection $\nabla$ on a finite Kasparov 
module $\mathcal{E}$, by sending 1 -forms $\delta_{u} a$ to $[D, a]$, one can associate with the universal connection $\nabla$ a $\sigma$-connection $\nabla_{D}$ on $\mathscr{E}$ for any spectral triple $\sigma=(\mathcal{A}, \mathscr{H}, D)$.

Let $p \in M_{n}(\mathcal{A})$ be a projection (i.e., a self-adjoint idempotent $(n \times n)$-matrix). Now we consider the right $\mathcal{A}$-module $p \mathcal{A}^{n}$. There is a canonical universal connection on $p \mathcal{A}^{n}$, which is given by the matrix $p \operatorname{diag}\left\{\delta_{u}, \ldots, \delta_{u}\right\}$ or simply $\nabla(p a)=$ $p \delta_{u}(p \boldsymbol{a})$ for all $\boldsymbol{a} \in \mathcal{A}^{n}$, and this connection is Hermitian.

Definition 4.2. A universal connection $\nabla$ on a pre-Hilbert right $\mathcal{A}$-module $S$ is said to be projectional if there is a unitary $\mathcal{A}$-isomorphism $\phi: S \rightarrow p \mathcal{A}^{n}$ for some $n$ and some projection $p$ such that $\nabla=\phi^{-1} \circ p \delta_{u} \circ \phi$.

A projectional universal connection on each finite projective pre-Hilbert $\mathcal{A}$-module is unique up to unitary $\mathcal{A}$-isomorphism. If $\mathcal{E}$ is a finite Kasparov $\mathscr{B}$-A-module admitting a universal connection $\nabla^{\mathcal{E}}$ and $\mathcal{F}$ is a finite Kasparov $\mathcal{C}$ - $\mathcal{B}$-module admitting a universal connection $\nabla^{\mathscr{F}}$, then there is a twisted universal connection $\nabla^{\mathscr{F}} \circ \nabla^{\mathcal{E}}$ defined in an obvious way on the finite Kasparov $\mathcal{C}$-A $-\mathcal{A}$-module $\mathcal{F} \otimes_{\mathcal{B}} \mathcal{E}$. Furthermore if $\nabla^{\mathcal{E}}$ and $\nabla^{\mathscr{F}}$ are projectional then so is $\nabla^{\mathscr{F}} \circ \nabla^{\mathcal{E}}$. This gives rise to a category - the category of noncommutative differential spaces NDG defined as follows. Objects in NDG consist of all unital pre- $C^{*}$ algebras. If $\mathcal{A}$ is a unital pre- $C^{*}$ algebra, we denote by $X_{\mathcal{A}}$ the corresponding object in NDG. Morphisms from $X_{\mathcal{A}}$ to $X_{\mathscr{B}}$ in NDG are isomorphism classes of finite Kasparov $\mathcal{B}$ - $\mathcal{A}$-modules with projectional universal connections. If $\mathcal{E}$ is an finite Kasparov $\mathcal{B}$ - $\mathcal{A}$-module with a projective universal connection $\nabla$, we denote by $(\mathcal{E}, \nabla)$ the corresponding morphism in $\operatorname{Hom}_{\mathrm{NDG}}\left(X_{\mathcal{A}}, X_{\mathcal{B}}\right)$.

Denote by $\operatorname{Sptr}^{0}(\mathcal{A})$ the set of unitary equivalence classes of even spectral triples for $\mathcal{A}$. The set $\operatorname{Sptr}^{0}(\mathcal{A})$ has an abelian monoid structure, the binary operation of which is the direct sum operation for spectral triples with algebra $\mathcal{A}$. Thus $\operatorname{Sptr}^{0}$ yields a functor from NDG to AbMonoid, the category of abelian monoids, given by

$$
X_{\mathcal{A}} \mapsto \operatorname{Sptr}^{0}(\mathcal{A}) \quad \text { and } \quad \operatorname{Sptr}^{0}((\mathcal{E}, \nabla)): \sigma \mapsto \sigma^{\mathcal{E}} \quad \text { for all } \sigma \in \operatorname{Sptr}^{0}(\mathcal{A}) \text {. }
$$

Remark 4.3. It is not difficult to extend morphisms of NDG to graded modules with super-connections in the sense of Quillen [23]; however, in this paper except Section 6, we only focus on finite Kasparov modules with trivial gradings. Baaj-Julg [2] established the theory of unbounded Kasparov modules and showed that every element in the bivariant KK-theory can be represented by an unbounded Kasparov module. It would be nice if morphisms of NDG could be enlarged to unbounded (even) Kasparov modules $(E, \mathcal{D}, \Gamma)$ with a grading $\Gamma$ and an appropriate universal connection $\nabla$, and thereby $D^{E, \mathcal{D}, \Gamma}\left(\xi \otimes_{\mathcal{A}} h\right)=\mathcal{D} \xi \otimes_{\mathcal{A}} h+\Gamma \xi \otimes_{\mathcal{A}} D h+\Gamma(\nabla \xi) h$. The notion of connection for bounded Kasparov modules introduced by ConnesSkandalis was well known, whereas theory of connection for unbounded Kasparov modules has been developed in a recent work by Mesland [22]. 
Definition 4.4. Two unital spectral triples $\sigma_{1}=\left(\mathcal{A}_{1}, \mathscr{H}_{1}, D_{1}\right)$ and $\sigma_{2}=\left(\mathcal{A}_{2}, \mathscr{H}_{2}\right.$, $D_{2}$ ) are said to be Morita equivalent if $\mathcal{A}_{1}$ and $\mathcal{A}_{2}$ are Morita equivalent as algebras and there is a finite Kasparov $\mathcal{A}_{2}-\mathcal{A}_{1}$-module $\mathcal{E}$ with a $\sigma_{1}$-connection such that $\mathscr{E}$ is an equivalence bimodule and $\sigma_{2}$ is unitarily equivalent to $\sigma_{1}^{\mathscr{E}}$.

Notice that the definition of Morita equivalence for spectral triples here, using $\sigma$ connections rather than the usual universal connections, will give rise to a symmetric relation:

Theorem 4.5. The Morita equivalence between spectral triples is an equivalence relation.

Proof. Reflexivity follows since $(\mathcal{A}, \mathcal{H}, D)$ is Morita equivalent to itself via the trivial connection $\nabla a=[D, a]$ on $\mathcal{A}$. Transitivity is straightforward by definition.

Symmetry: Suppose that $\left(\mathscr{B}, \mathscr{H}^{\mathcal{E}}, D^{\mathcal{E}}\right)$ is Morita equivalent to $\sigma=(\mathcal{A}, \mathscr{H}, D)$ via a $\sigma$-connection $\nabla$ on $\mathcal{E}$. Let $\mathscr{F}=\mathcal{E}^{*}$. Define $\nabla^{\mathscr{F}}: \mathscr{F} \rightarrow \mathscr{F} \otimes_{\mathcal{B}} B\left(\mathscr{H}^{\mathcal{E}}\right)$ by

$$
\left(\nabla^{\mathscr{F}} f\right)\left(\xi \otimes_{\mathcal{A}} h\right)=-f(\nabla \xi) \otimes_{\mathcal{A}} h+[D, f \xi] h \quad \text { for all } f \in \mathscr{F}, \xi \in \mathcal{E}, h \in \mathcal{H} .
$$

Here we use a map $\mathscr{H}^{\mathcal{E}} \rightarrow \mathscr{H}$ to represent an element in $\mathscr{F} \otimes_{\mathcal{B}} B\left(\mathscr{H}^{\mathcal{E}}\right)$. One can verify that $\nabla^{\mathscr{F}}$ is a $\left(\mathscr{B}, \mathscr{H}^{\mathcal{E}}, D^{\mathcal{E}}\right)$-connection. Now we check $\left(D^{\mathcal{E}}\right)^{\mathscr{F}}=D$ as follows,

$$
\begin{aligned}
\left(D^{\mathcal{E}}\right)^{\mathcal{F}}\left(f \otimes_{\mathcal{B}} \xi \otimes_{\mathcal{A}} h\right) & =f \otimes_{\mathcal{B}} D^{\mathcal{E}}\left(\xi \otimes_{\mathcal{A}} h\right)+\left(\nabla^{\mathcal{F}} f\right)\left(\xi \otimes_{\mathcal{A}} h\right) \\
& =f \otimes_{\mathcal{B}} \xi D h+f(\nabla \xi) h+\left(\nabla^{\mathscr{F}} f\right)\left(\xi \otimes_{\mathcal{A}} h\right) \\
& =f \xi D h+f(\nabla \xi) h-f(\nabla \xi) h+[D, f \xi] h \\
& =D(f \xi h) .
\end{aligned}
$$

(An alternative way to prove the symmetry property is using bounded perturbations of the $\sigma$-connection and $\sigma^{\mathcal{E}}$-connection that are associated to projectional universal connections.) In conclusion, Morita equivalence of spectral triples is an equivalence relation.

Remark 4.6. There are at least three possible spaces of "connections" for spectral triples:

(i) the space of $\sigma$-connections;

(ii) the space of 1-form connections, i.e., connections induced from universal connections, which is a subspace of type (i);

(iii) the space of connections induced from projectional universal connections, which is a subspace of type (ii).

There are several reasons not restricting ourselves to the 1-form connections when trying to define Morita relation between spectral triples: 
(i) The Morita relation defined using 1-form connections is not symmetric.

(ii) For the $Z_{2}$-graded cases (see Section 6), one needs to use super-connections which are in general high order form connections [3].

(iii) The Morita relation defined using 1-form connections does not include all bounded perturbations of Dirac operators.

As a special case, $\mathcal{A}$ is Morita equivalent to itself via $\mathscr{E}=\mathcal{A}$. Using universal connections on $\mathcal{A}$, one can construct new spectral triples which are called inner fluctuations of spectral triples [9]. Morita equivalence of spectral triples with common algebra $\mathcal{A}$ and pre-Hilbert space $\mathcal{H}$ is exactly bounded perturbation of Dirac operators. In fact, $(\mathcal{A}, \mathcal{H}, D)$ is Morita equivalent to $(\mathcal{A}, \mathcal{H}, D+B)$ for any bounded self-adjoint operator $B$ (or bounded odd self-adjoint operator $B$ in the even case) through the bimodule $\mathcal{A}$ and the $(\mathcal{A}, \mathscr{H}, D)$-connection $\nabla$ given by $\nabla(1):=B$. Conversely, $(\mathcal{A}, \mathscr{H}, D+B)$ is Morita equivalent to $(\mathscr{A}, \mathscr{H}, D)$ through the $(\mathscr{A}, \mathscr{H}, D+B)$-connection $\nabla^{*}$ given by $\nabla^{*}(1)=-B$. This is not the case for inner fluctuation of spectral triples, as the latter is generally not an equivalence relation when $\mathcal{A}$ is noncommutative. In general, if we confine ourselves to equivalence bimodules with universal connections, the symmetry property in the above proof does not hold. However, if we restrict the universal connections on equivalence bimodules to the projectional ones, the symmetry property holds again. Unfortunately, Levi-Civita connections are not projectional connections.

Suppose that $(\mathscr{E}, \nabla)$ is a morphism in $\operatorname{Hom}_{\mathrm{NDG}}\left(X_{\mathcal{A}}, X_{\mathscr{B}}\right)$. There is a free right $\mathcal{A}$-module $\mathcal{A}^{m}$ and a projection $p$ in $\mathrm{M}_{m}(\mathcal{A})$ such that $\mathcal{E} \cong p \mathcal{A}^{m}$. Let $\left\{e_{1}, \ldots, e_{m}\right\}$ be the standard generators of $\mathcal{A}^{m}$. For each $b$ in $\mathscr{B}$, one can find a matrix $\alpha(b)$ in $p \mathrm{M}_{m}(\mathcal{A}) p$ such that $b p e_{i}=\sum_{j} e_{j} \alpha_{j i}(b)$.

The K-theory, Hochschild (co)homology and (periodic) cyclic (co)homology are all functors on the category NDG. For instance, suppose that $\left(b_{0}, \ldots, b_{n}\right)$ is a Hochschild $n$-cycle representing an element $\Phi \in \mathrm{HH}_{*}(\mathscr{B})$. Then $\mathcal{E}(\Phi) \in \mathrm{HH}_{*}(\mathscr{A})$ is the Hochschild $n$-cycle given by the Dennis trace map

$$
\operatorname{tr}\left(\alpha\left(b_{0}\right) \otimes \cdots \otimes \alpha\left(b_{n}\right)\right)=\sum_{i_{0}, \ldots, i_{n}}\left(\alpha_{i_{0} i_{1}}\left(b_{0}\right), \alpha_{i_{1} i_{2}}\left(b_{1}\right), \ldots, \alpha_{i_{n} i_{0}}\left(b_{n}\right)\right) .
$$

Note that $\alpha$ depends on the choice of the isomorphism $\mathcal{E} \cong p \mathcal{A}^{m}$; however, it does induce the well-defined morphisms $\mathcal{E}: \mathrm{HH}_{*}(B) \rightarrow \mathrm{HH}_{*}(\mathcal{A})$.

Furthermore, the Connes-Chern characters [6] are natural transformations from the K-theory functor $X_{\mathscr{A}} \mapsto K_{0}(\mathcal{A})$ to the periodic cyclic homology functor $X_{\mathscr{A}} \mapsto$ $\mathrm{HP}_{0}(P)$ and from functors $X_{\mathcal{A}} \mapsto \operatorname{Sptr}^{0}(\mathcal{A})$ and $X_{\mathcal{A}} \mapsto K^{0}(\overline{\mathcal{A}})$ to the periodic cyclic cohomology functor $X_{\mathscr{A}} \mapsto \operatorname{HP}^{0}(\mathcal{A})$. The naturalness of Connes-Chern characters 
is illustrated in the following commutative diagrams
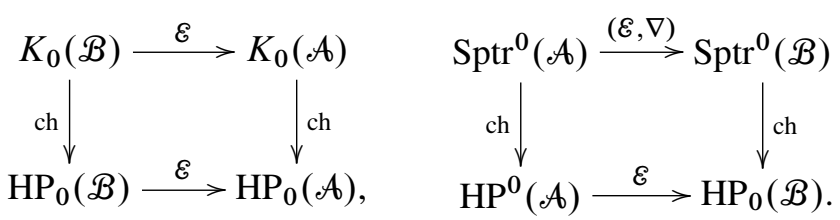

Proposition 4.7. The diagrams
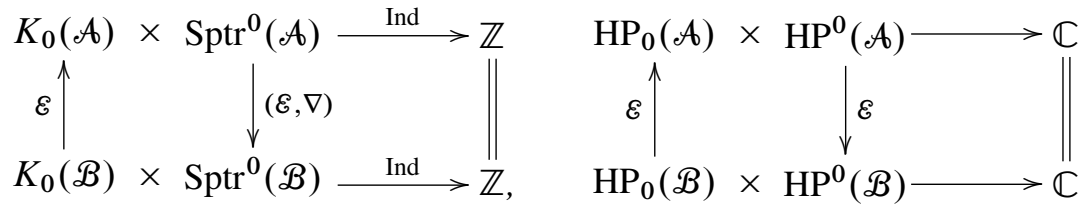

and

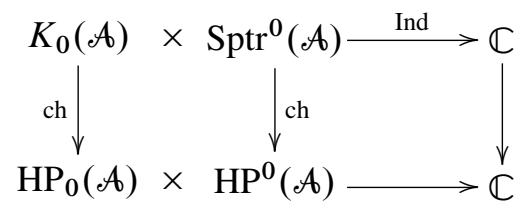

commute.

\section{Gluing local spin structures on Riemannian manifolds via Morita equivalence}

We can apply the above theory to spectral triples on Riemannian manifolds. By gluing local pieces of spectral triples via Morita equivalence, we construct a socalled projective spectral triple, the Dirac operator of which was defined in a formal sense by Mathai-Melrose-Singer [20].

Let $X$ be a closed oriented Riemannian manifold of dimension $n$. Suppose that $X$ is spin or $\operatorname{spin}^{c}$. Let $\mathrm{Cl}_{n}$ denote the complex Clifford algebra of $\mathbb{R}^{n}$. In this paper we use the following convention for the definition of Clifford algebras:

$$
\left.\mathrm{Cl}_{n}:=\left\langle u \in \mathbb{R}^{n}\right| u v+v u=-2(u, v) \text { for all } u, v \in \mathbb{R}^{n}\right\rangle .
$$

Decomposed by the parity of the degree, $\mathrm{Cl}_{n}=\mathrm{Cl}_{n}^{0} \oplus \mathrm{Cl}_{n}^{1}$. Write

$$
B_{n}=\left\{\begin{array}{l}
\mathrm{Cl}_{n} \cong \mathrm{M}_{2^{m}}(\mathbb{C}) \quad \text { when } n=2 m \text { is even, } \\
\mathrm{Cl}_{n}^{0} \cong \mathrm{M}_{2^{m}}(\mathbb{C}) \quad \text { when } n=2 m+1 \text { is odd, }
\end{array}\right.
$$

and

$$
B_{x}=\left\{\begin{array}{l}
\mathrm{Cl}\left(T_{x}^{*} X\right) \\
\mathrm{Cl}^{0}\left(T_{x}^{*} X\right)
\end{array}\right.
$$


Denote by $\mathrm{Cl}(X)$ and $B(X)$ the vector bundles over $X$ whose fibers at a point $x \in X$ are $\mathrm{Cl}\left(T_{x}^{*} X\right)$ and $B_{x}$. Let $S_{n}=\mathbb{C}^{2^{m}}$ be the standard spinor vector space, fix a specific isomorphism

$$
c: B_{n} \rightarrow \operatorname{End}_{\mathbb{C}}\left(S_{n}\right) .
$$

Let $\omega_{n}=i^{\left[\frac{n+1}{2}\right]} e_{1} \ldots e_{n} \in \mathrm{Cl}_{n}$. When $n$ is odd, $B_{n}=\omega_{n} \mathrm{Cl}_{n}^{1}$. This indicates a homomorphism $c: \mathrm{Cl}_{n} \rightarrow \operatorname{End}_{\mathbb{C}}\left(S_{n}\right)$. When $n$ is even, $S_{n}=S_{n+} \oplus S_{n-}$ is graded by the eigenspaces of $\omega_{n}$ which are invariant under the action of $\operatorname{Spin}(n)$.

Let $P_{\mathrm{Fr}}(X)$ and $P_{\mathrm{Spin}(\mathrm{c})}(X)$ denote the orthonormal oriented frame bundle and the principal $\operatorname{Spin}(n)$ or $\operatorname{Spin}^{\mathrm{c}}(n)$-bundle over $X$ respectively. Then

$$
\mathrm{Cl}(X)=P_{\mathrm{Fr}}(X) \times_{\mathrm{SO}(n)} \mathrm{Cl}_{n}=P_{\mathrm{Spin}(c)}(X) \times_{\mathrm{Ad}} \mathrm{Cl}_{n} .
$$

The spinor bundle over $X$ is the associated $\operatorname{Spin}\left({ }^{c}\right)(n)$-bundle

$$
S_{X}=P_{\operatorname{Spin}(\mathfrak{c})}(X) \times_{c} S_{n} .
$$

The Clifford algebra bundle acts naturally on the spinor bundle, $c: \mathrm{Cl}(X) \times_{X} S_{X} \rightarrow$ $S_{X}$, which is given by

$$
(p, \xi) \times(p, s) \mapsto(p, c(\xi) s) \quad \text { for all } p \in P_{\operatorname{Spin}(c)}(X), \xi \in \mathrm{Cl}_{n}, s \in S_{n} .
$$

When $n$ is even, $\omega_{n}$ induces a grading operator $\omega$ on $S_{X}=S_{X+} \oplus S_{X-}$.

Denote by $\not D$ the Dirac operator on $S_{X}$. Let $E$ be a Hermitian vector bundle over $M$ with a Hermitian connection $\nabla^{E}$. Let $\mathcal{A}=C^{\infty}(X), \mathscr{B}=\Gamma(B(X)), \mathcal{E}=\Gamma(E)$. Then the well-known spin spectral triple

$$
(\mathcal{A}, \mathscr{H}, D)=\left(C^{\infty}(X), \Gamma\left(S_{X}\right), \not D\right)
$$

is Morita equivalent to the spectral triple with a noncommutative algebra

$$
\left(\mathscr{B}, \mathscr{H}^{\mathcal{E}}, D^{\mathcal{E}}\right)=\left(\Gamma(\operatorname{End}(E)), \Gamma\left(E \otimes S_{X}\right) \not D^{E}\right),
$$

via the finite Kasparov module $\mathcal{E}$ with $(\mathcal{A}, \mathcal{H}, D)$-connection associated with $\nabla^{E}$. Here $\not D^{E}$ denotes the twisted Dirac operator on the vector bundle $E \otimes S_{X}$.

Now interesting things happen when a manifold has no $\operatorname{spin}^{\mathrm{c}}$ structure: The spinor bundle does not exist, and neither does the spin spectral triple. However, as constructed later in this section, for any closed oriented Riemannian manifold, not necessarily $\operatorname{spin}^{\mathrm{c}}$, there is a canonical noncommutative spectral triple:

Definition 5.1. The projective spectral triple of a closed oriented Riemannian manifold $M$ is defined to be

$$
\left(\mathcal{A}_{W_{3}}, \mathcal{H}_{W_{3}}, D_{W_{3}}\right):=\left(\Gamma(B(M)),(1+*) \Omega(M),\left(d-d^{*}\right)(-1)^{\mathrm{deg}}\right)
$$

if $M$ is odd-dimensional, and

$$
\left(\mathcal{A}_{W_{3}}, \mathcal{H}_{W_{3}}, D_{W_{3}}, \gamma_{W_{3}}\right):=\left(\Gamma(B(M)), \Omega(M),\left(d-d^{*}\right)(-1)^{\operatorname{deg}}, *(-1)^{\frac{\operatorname{deg}(\operatorname{deg}+1)}{2}-\frac{n}{4}}\right)
$$

if $M$ is even-dimensional. 
We can consider that projective spectral triples are obtained by gluing local spin spectral triples in the following way:

Let $M$ be a closed oriented Riemannian manifold of dimension $n$, not necessarily $\operatorname{spin}^{\mathrm{c}}$. Let $\left\{U_{i}\right\}$ be a good covering of $M$. Then on each local piece we have the principal $\operatorname{Spin}(n)$-bundle $P_{i}=P_{\mathrm{Spin}}\left(U_{i}\right)$, the associated spinor bundle $S_{i}=S_{U_{i}}$, the spin connection $\nabla_{i}$, and the Dirac operator $\not_{i}$. Over each intersection $U_{i j}=$ $U_{i} \cap U_{j} \neq \emptyset$, there is up to $\pm 1 \in \operatorname{Spin}(n)$ a unique homeomorphism of principal bundles

such that

$$
\alpha_{i j}:\left.\left.P_{i}\right|_{U_{i j}} \rightarrow P_{j}\right|_{U_{i j}}
$$

$$
\alpha_{i j}\left(p_{i} g\right)=\alpha_{i j}\left(p_{i}\right) g \text { for all }\left.p_{i} \in P_{i}\right|_{U_{i j}}, g \in \operatorname{Spin}(n) .
$$

This homeomorphism induces up to \pm 1 a Clifford module homomorphism $\alpha_{i j}:\left.\left.S_{i}\right|_{U_{i j}} \rightarrow S_{j}\right|_{U_{i j}}$,

$$
\alpha_{i j}(p, s)=\left(\alpha_{i j}(p), s\right) \quad \text { for all }\left.p \in P_{i}\right|_{U_{i j}}, s \in S_{n} .
$$

For each triple overlap $U_{i j k}=U_{i} \cap U_{j} \cap U_{k} \neq \emptyset$, write

$$
\sigma_{i j k}=\left.\alpha_{k i} \circ \alpha_{j k} \circ \alpha_{i j}\right|_{U_{i j k}} .
$$

Then $\left\{\sigma_{i j k}\right\}$ represents a Čech cocycle in $\check{H}^{2}\left(M, \mathbb{Z}_{2}\right)$, and the corresponding singular class $w_{2}(M) \in H^{2}\left(M, \mathbb{Z}_{2}\right)$ is the second Stiefel-Whitney class. Let $L_{i j}=$ $\operatorname{Hom}_{\mathrm{Cl}\left(U_{i j}\right)}\left(\left.S_{i}\right|_{U_{i j}},\left.S_{j}\right|_{U_{i j}}\right)$ denote the line bundle of the Clifford module homomorphisms of $S_{i}$ and $S_{j}$ over $U_{i j}$. Then $\alpha_{i j}$ is the canonical section of $L_{i j}$. $\left\{L_{i j}\right\}$ forms a gerbe of line bundles over $M$ characterized by the Dixmier-Douady class $\delta(B(M))=W_{3}(M)$ (the third integral Stiefel-Whitney class). We refer to [4] for (twisted) K-theory of bundle gerbes.

If $M$ is spin, then for each $U_{i j}$ there is $\beta_{i j} \in \mathbb{Z}_{2}$ such that $\left\{\beta_{i j} \alpha_{i j}\right\}$ satisfies the cocycle condition. That means the local spinor bundles $S_{i j}$ can be glued together as a global spinor bundle via the Clifford module isomorphisms $\beta_{i j} \alpha_{i j}$. The difference between two different choices of such collection $\left\{\beta_{i j}\right\}$ is a cocycle in $\breve{H}^{1}\left(M, \mathbb{Z}_{2}\right)$ which parameterizes distinct spin structures on $M$.

If $M$ is $\operatorname{spin}^{\mathrm{c}}$, then for each $U_{i j}$ there is $\beta_{i j} \in C^{\infty}\left(U_{i j}, U(1)\right)$ such that $\left\{\beta_{i j} \alpha_{i j}\right\}$ satisfies the cocycle condition. That means that the local spinor bundles $S_{i j}$ can be glued together as a global spinor bundle via the Clifford module isomorphisms $\beta_{i j} \alpha_{i j}$. The collection $\left\{\beta_{i j}^{2}\right\}$ also satisfies the cocycle condition, and the Čech cocycle $\left\{\beta_{i j}^{2}\right\} \in \breve{H}^{1}(M, U(1))$ corresponds to the canonical line bundle $\mathcal{L}$ of a spin ${ }^{\mathrm{c}}$ structure. The difference between two different choices of such collection $\left\{\beta_{i j}\right\}$ is a cocycle in $\breve{H}^{1}(M, U(1))$ which parameterizes distinct spinor bundles on $M$.

Denote by $C^{\infty}\left(\bar{U}_{i}\right)$ the space of smooth functions on $U_{i}$ that can be extended to a smooth function on a small open neighborhood $V_{i}$ of $\bar{U}_{i}$, and likewise denote by 
$\Gamma\left(\bar{U}_{i}, \cdot\right)$ for extendable smooth sections. Take $\varepsilon_{i}=\Gamma\left(\bar{U}_{i}, S_{i}\right)$, then the "local spin spectral triple"

$$
\left(\mathcal{A}_{i}, \mathscr{H}_{i}, D_{i}\right)=\left(C^{\infty}\left(\bar{U}_{i}\right), \Gamma\left(\bar{U}_{i}, S_{i}\right), \not D_{i}\right)
$$

is Morita equivalent to the local spectral triple

$$
\left(\mathcal{B}_{i}, \mathscr{H}_{i}^{\mathcal{E}_{i}}, D_{i}^{\mathcal{E}_{i}}\right)=\left(\Gamma\left(\bar{U}_{i}, B\left(U_{i}\right)\right), \Gamma\left(\bar{U}_{i}, S_{i} \otimes S_{i}\right), \not D_{i}^{S_{i}}\right) .
$$

Remark 5.2. We use here the triple (5) to formulate the spin structure of the open subset $U_{i}$, but it is by definition not a spectral triple, for the compact resolvent condition fails. However, it can naturally act on the relative K-theory for the pair of spaces $\left(V_{i}, U_{i}\right)$ or $\left(Y, \iota\left(U_{i}\right)\right)$ to get an index, where $Y$ is any compact Riemannian spin manifold that admits an isometric embedding $\iota: V_{i} \rightarrow Y$. By the excision property, the choice of $V_{i}$ is irrelevant. In this sense the triple (5) represents a relative $\mathrm{K}$-cycle. One may also think of the standard treatment using the nonunital spectral triple $\left(C_{c}^{\infty}\left(U_{i}\right), L^{2}\left(U_{i}, S_{i}\right), \not D_{i}\right)$ with the algebra of smooth functions with compact support. See Gayral-Gracia-Bondía-Iochum-Schücker-Várilly [14] for a set of axioms for nonunital spectral triples which is proposed to set up the notion of noncompact noncommutative spin manifolds. This, however, will cause some subtleties when considering Morita equivalence and the smoothness condition.

Because the collection of maps $\left\{\alpha_{i j} \otimes \alpha_{i j}\right\}$ satisfies the cocycle condition, the vector bundles $S_{i} \otimes S_{i}$ and Dirac operators $D_{i}^{\mathcal{E}_{i}}$ can be glued together to form a vector bundle $N$ over $M$ and a Dirac operator $D_{N}$ on $N$, so that $\left(\left.\mathscr{B}\right|_{U_{i}},\left.\Gamma(M, N)\right|_{U_{i}},\left.D_{N}\right|_{U_{i}}\right)$ are unitarily equivalent to $\left(\mathscr{B}_{i}, \mathscr{H}_{i}^{\mathcal{E}_{i}}, D_{i}^{\mathcal{E}_{i}}\right)$, where $\mathscr{B}=\Gamma(M, B(M))$. Thus we succeed to construct a globally well-defined spectral triple $\left(\mathscr{B}, \Gamma(N), D_{N}\right)$ on $M$.

Proposition 5.3. The global vector bundle $N$ is isomorphic to $B(M)$, and $\Gamma(N)$ is isomorphic to $\Gamma(B(M))$ as both $\Gamma(B(M))$-modules and pre-Hilbert spaces.

Proof. Let $S_{n}^{*}$ be the dual vector space of the standard spinor vector space $S_{n}$. We endow $S_{n}^{*}$ with a left $B_{n}$-module structure by $\gamma^{*}(b) f_{x}:=f_{\bar{b} x}=\left(x, \bar{b}^{*}\right.$.) for all $x \in S_{n}, b \in B_{n}$, where $\bar{b}$ is the complex conjugate of $b$ and $b^{*}$ is the adjoint of $b \in B_{n} \cong \mathrm{M}_{2^{m}}(\mathbb{C})$. Since $B_{n}$ is a simple algebra, up to a scalar there is a unique $B_{n}$-module isomorphism from $S_{n}$ to $S_{n}^{*}$. We fix one specific unitary $B_{n}$-module isomorphism $T_{n}: S_{n} \rightarrow S_{n}^{*}$. Then $T_{n}$ induces a Clifford module isomorphism of bundles $T: S_{i} \rightarrow S_{i}^{*}$ given by $(p, s) \mapsto\left(p, T_{n} s\right)$ for all $p \in P_{i}, s \in S_{n}$. Let $S_{i}^{*}=P_{i} \times_{\gamma^{*}} S_{n}^{*}$ and let $\alpha_{i j}^{*}: S_{i}^{*} \rightarrow S_{j}^{*}$ denote the Clifford module isomorphism given by $\left.\alpha_{i j}^{*}(p, f)\right)=\left(\alpha_{i j} p, f\right)$ for all $f \in S_{i}^{*}$. The mappings $T$ on $U_{i}$ and $U_{j}$ are 
compatible, namely the diagram below commutes on $U_{i j}$ :

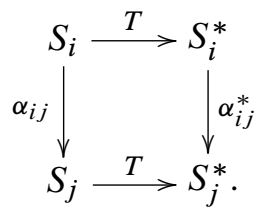

Then one can glue the bundles $S_{i}^{*} \otimes S_{i} \cong \operatorname{End}_{\mathbb{C}}\left(S_{i}^{*}\right)$ together as a global bundle $B(M)$ via the maps $\alpha_{i j}^{*} \otimes \alpha_{i j}$, and $T$ induces an isomorphism from $N$ to $B(M)$. Then it is easy to verify the proposition.

Corollary 5.4. In the case that $M$ is odd-dimensional, one can take the bundle $N=(1+*) \bigwedge^{*}\left(T_{\mathbb{C}}^{*} M\right)$. Then the spectral triple $\left(\mathscr{B}, \Gamma(N), D_{N}\right)$ is the projective spectral triple

$$
\left(\mathcal{A}_{W_{3}}, \mathscr{H}_{W_{3}}, D_{W_{3}}\right)=\left(\mathscr{B},(1+*) \Omega(M),\left(d-d^{*}\right)(-1)^{\mathrm{deg}}\right) .
$$

When $M$ is even-dimensional, take $N=\wedge^{*}\left(T_{\mathbb{C}}^{*} M\right)$. Then the spectral triple $\left(\mathscr{B}, \Gamma(N), D_{N}\right)$ with the grading on $\Gamma(N)$ obtained from the grading on $S_{i}$ is the even projective spectral triple

$$
\left(\mathcal{A}_{W_{3}}, \mathscr{H}_{W_{3}}, D_{W_{3}}, \gamma_{W_{3}}\right)=\left(\mathcal{B}, \Omega(M),\left(d-d^{*}\right)(-1)^{\mathrm{deg}}, *(-1)^{\operatorname{deg}(\operatorname{deg}+1) / 2-n / 4}\right) \text {. }
$$

Its center $\left(\mathcal{A}_{,} \mathscr{H}_{W_{3}}, D_{W_{3}}, \gamma_{W_{3}}\right)$ is unitarily equivalent to the spectral triple for Hirzebruch signature. For any $a \in \mathcal{A}$, the commutator $\left[D_{W_{3}}, a\right]$ is just the right Clifford action of da on $\mathscr{H}_{W_{3}}$.

Theorem 5.5. If $M$ is $\operatorname{spin}^{\mathrm{c}}$, the projective spectral triple on $M$ is Morita equivalent to the spin spectral triple.

Proof. Let $S$ be the global spinor bundle over $M$. There exist local half line bundles $\mathcal{L}_{i}^{1 / 2}$ such that $\mathcal{L}_{i}^{1 / 2}$ are characterized by $\left\{\beta_{i j}\right\}$ as local transition functions: $\mathcal{L}_{i}^{1 / 2} \otimes$ $\mathcal{L}_{i}^{1 / 2}=\left.\mathcal{L}\right|_{U_{i}}$ and $\left.S\right|_{U_{i}}=\mathcal{L}_{i}^{1 / 2} \otimes S_{i}$. There also exists a hermitian connection $\nabla_{i}^{\prime}$ on $\mathcal{L}_{i}^{1 / 2}$ such that for the $\operatorname{spin}^{c}$-connection we have $\left.\nabla\right|_{U_{i}}=1 \otimes \nabla_{i}+\nabla_{i}^{\prime} \otimes 1$, where $\nabla_{i}$ is the spin-connection on $S_{i}$. Then follows the Morita equivalence of the spectral triples

$$
\begin{aligned}
\left(C^{\infty}(M), \Gamma(M, S), \not D\right) & =(\mathscr{A}, \mathcal{H}, D) \\
& \sim\left(\mathcal{A}, \mathscr{H}^{\mathcal{L}}{ }^{-1}, D^{\mathcal{L}}{ }^{-1}\right) \\
& \sim\left(\mathscr{B},\left(\mathscr{H}^{\mathcal{L}^{-1}}\right)^{S},\left(D^{\mathcal{L}^{-1}}\right)^{S}\right) \\
& =\left(\mathcal{A}_{W_{3}}, \mathscr{H}_{W_{3}}, D_{W_{3}}\right) .
\end{aligned}
$$

We see that the projective spectral triple is defined for any closed oriented Riemannian manifold regardless of whether the manifold is $\operatorname{spin}^{\mathrm{c}}$ or not. The projective spectral triple depends only on the metric and orientation of $M$ and does not depend on the choice of the local spinor bundles $S_{i}$. 


\section{Kasparov's spectral triple}

In the Introduction we mentioned that Kasparov's fundamental class, the Dirac element [17], is essentially an even spectral triple with a $\mathbb{Z}_{2}$-graded underlying algebra, and we claimed that the projective spectral triple is in fact Morita equivalent to Kasparov's spectral triple. In this section we see in detail how these two spectral triples are related by Morita equivalence.

It is very easy to make sense of even spectral triples with $\mathbb{Z}_{2}$-graded algebras and Morita equivalence between them. Replacing all the commutator relations for even spectral triples by graded commutator relations, the definition of even spectral triples with $\mathbb{Z}_{2}$-graded algebras will be the same as that of even spectral triples with ungraded algebras. Similarly, we can generalize $\sigma$-connections to $\sigma$-super-connections. To be compatible with graded commutator relations, $\sigma$-super-connections are required to be odd operators. Two $\mathbb{Z}_{2}$-graded algebras $\mathcal{A}_{1}$ and $\mathcal{A}_{2}$ are Morita equivalent through a $\mathbb{Z}_{2}$-graded $\mathcal{A}_{2}-\mathcal{A}_{1}$-bimodule $\mathcal{E}$ with grading $\gamma_{21}$ if $\mathcal{A}_{1}$ and $\mathcal{A}_{2}$ as ungraded algebras are Morita equivalent through $\mathcal{E}$ as an ungraded module and $\mathcal{A}_{2} \cong \operatorname{End}_{\mathcal{A}_{1}}(\mathscr{E})$ as $\mathbb{Z}_{2}$-graded algebras. Suppose that $\sigma=\left(\mathcal{A}_{1}, \mathcal{H}_{1}, D_{1}, \gamma_{1}\right)$ is an even spectral triple with $\mathbb{Z}_{2}$-graded algebra $\mathcal{A}_{1}, \mathcal{E}$ is a $\mathbb{Z}_{2}$-graded $\mathcal{A}_{2}-\mathcal{A}_{1}$-bimodule with grading $\gamma_{21}$, and $\nabla$ is a $\sigma$-super-connection on $\mathcal{E}$. We define

$$
\begin{aligned}
D_{1}^{\mathcal{E}}\left(\xi \otimes_{\mathcal{A}_{1}} h\right) & =\gamma_{21} \xi \otimes_{\mathcal{A}_{1}} D_{1} h+\gamma_{21}(\nabla \xi) h \quad \text { for all } h \in \mathscr{H}_{1}, \xi \in \mathcal{E}, \\
\gamma_{1}^{\mathcal{E}} & =\gamma_{21} \otimes_{\mathcal{A}_{1}} \gamma_{1} .
\end{aligned}
$$

Then we can straightforwardly extend the notion of Morita equivalence of spectral triples to the case of even spectral triples with $\mathbb{Z}_{2}$-graded algebras.

Recall that the projective spectral triple for an even-dimensional closed Riemannian manifold $M$ is an even spectral triple

$$
\left(\mathcal{A}_{1}, \mathscr{H}_{1}, D_{1}, \gamma_{1}\right)=\left(\mathscr{B}, \Omega(M),\left(d-d^{*}\right)(-1)^{\operatorname{deg}}, *(-1)^{\operatorname{deg}(\operatorname{deg}+1) / 2-\operatorname{dim} M / 4}\right),
$$

where $\mathscr{B}$ is the algebra of smooth sections of the Clifford bundle of $M$ with trivial grading.

We define a variant of Kasparov's spectral triple to be the even spectral triple with a $\mathbb{Z}_{2}$-graded algebra

$$
\left(\mathcal{A}_{2}, \mathscr{H}_{2}, D_{2}, \gamma_{2}\right)=\left(\mathscr{B}_{\mathrm{gr}}, \Omega(M), \sqrt{-1}\left(d-d^{*}\right),(-1)^{\mathrm{deg}}\right),
$$

where $\mathscr{B}_{\mathrm{gr}}$ is the algebra of smooth sections of the Clifford bundle of $M$ graded by the degree of its elements.

Proposition 6.1. $\left(\mathscr{A}_{1}, \mathscr{H}_{1}, D_{1}, \gamma_{1}\right)$ and $\left(\mathscr{A}_{2}, \mathscr{H}_{2}, D_{2}, \gamma_{2}\right)$ are Morita equivalent.

Proof. First note that $\left(\mathscr{A}_{1}, \mathscr{H}_{1}, D_{1}, \gamma_{1}\right)$ is unitarily equivalent to $\left(\mathcal{A}_{1}, \mathscr{H}_{1}, D_{1}^{\prime}, \gamma_{1}\right)$, where $D_{1}^{\prime}=\sqrt{-1} D_{1} \gamma_{1}$. Then the Morita equivalence between $\left(\mathcal{A}_{1}, \mathscr{H}_{1}, D_{1}, \gamma_{1}\right)$ 
and $\left(\mathcal{A}_{2}, \mathscr{H}_{2}, D_{2}, \gamma_{2}\right)$ will be given by a graded $\mathcal{A}_{2}-\mathcal{A}_{1}$-bimodule $\mathcal{E}_{21}$ with grading $\gamma_{21}$, together with a super-connection $\hat{\nabla}$ on $\varepsilon_{21}$, such that

$$
\left(\mathscr{A}_{2}, \mathscr{H}_{2}, D_{2}, \gamma_{2}\right)=\left(\operatorname{End}_{\mathcal{A}_{1}}\left(\mathcal{E}_{21}\right), \mathcal{E}_{21} \otimes_{\mathcal{A}_{1}} \mathscr{H}_{1}, D_{1}^{\prime \mathcal{E}_{21}}, \gamma_{21} \otimes_{\mathcal{A}_{1}} \gamma_{1}\right) .
$$

The construction of $\left(\mathcal{E}_{21}, \hat{\nabla}\right)$ is as follows.

Denote by $c_{\mathrm{L}}$ and $c_{\mathrm{R}}$ the left and right Clifford action of $\mathcal{B}$ on $\Omega(M)$ respectively. $\mathscr{B}$ is a complex algebra generated by $\Gamma\left(M, T_{\mathbb{R}}^{*} M\right)$. For any $\alpha \in \Gamma\left(M, T_{\mathbb{R}}^{*} M\right)$ and $\omega \in \Omega^{k}(M)$, we have

$$
\begin{aligned}
& c_{\mathrm{L}}(\alpha) \omega=\alpha \wedge \omega-\iota(\alpha) \omega, \\
& c_{\mathrm{R}}(\alpha) \omega=(-1)^{k}(\alpha \wedge \omega+\iota(\alpha) \omega),
\end{aligned}
$$

where $\iota$ is the contraction determined by the Riemannian metric $g$. Observe that $c_{\mathrm{L}}(\alpha) c_{\mathrm{R}}(\beta)=c_{\mathrm{R}}(\beta) c_{\mathrm{L}}(\alpha)$ and that

$$
\begin{aligned}
& *(a \wedge \omega)=(-1)^{k} \iota(\alpha)(* \omega), \\
& *(\iota(\alpha) \omega)=(-1)^{k+1} \alpha \wedge(* \omega) .
\end{aligned}
$$

Let $\mathcal{E}_{21}:=\Omega(M)$ be the $\mathscr{B}_{\mathrm{gr}}-\mathcal{B}$-bimodule with actions of $\mathscr{B}_{\mathrm{gr}}$ and $\mathscr{B}$ being the left and right Clifford action respectively. Now we consider three different gradings $\gamma_{1}, \gamma_{21}$ and $\gamma_{2}$ on $\Omega(M)$. We define

$$
\gamma_{21}(\omega):=(-1)^{k(k-1) / 2-n / 4} * \omega \text { for all } \omega \in \Omega^{k}(M),
$$

where $n=\operatorname{dim} M$ is an even number. One can easily check that $\gamma_{1}^{2}=\gamma_{2}^{2}=\gamma_{21}^{2}=$ id. By (6), (7), (8), (9) we have

$$
\begin{aligned}
c_{\mathrm{L}}(\alpha) \gamma_{1}(\omega) & =\gamma_{1}\left(c_{\mathrm{L}}(\alpha) \omega\right), \\
c_{\mathrm{R}}(\alpha) \gamma_{21}(\omega) & =\gamma_{21}\left(c_{\mathrm{R}}(\alpha) \omega\right) .
\end{aligned}
$$

We consider $\Omega(M) \stackrel{\cong}{\Longrightarrow} \mathcal{E}_{21} \otimes_{\mathcal{B}} \mathscr{H}_{1}$ via $\omega \mapsto \omega \otimes_{\mathcal{B}} 1$. This is an isomorphism of left $\mathscr{B}$-modules. By (10), (11), $\gamma_{21} \otimes_{\mathcal{B}} \gamma_{1}$ is well defined. Hence,

$$
\begin{aligned}
\left(\gamma_{21} \otimes_{\mathcal{B}} \gamma_{1}\right) \omega & =\gamma_{21}(\omega) \otimes_{\mathcal{B}} \gamma_{1}(1) \\
& =(-1)^{k(k-1) / 2-n / 2}(* \omega) \otimes_{\mathcal{B}}\left(e_{1} \wedge \ldots \wedge e_{n}\right) \\
& =(-1)^{k(k-1) / 2-n / 2}(* \omega) \otimes_{\mathcal{B}} c_{\mathrm{L}}\left(e_{1}\right) \ldots c_{\mathrm{L}}\left(e_{n}\right) 1 \\
& =(-1)^{k(k-1) / 2-n / 2} c_{\mathrm{R}}\left(e_{n}\right) \ldots c_{\mathrm{R}}\left(e_{1}\right)(* \omega) \otimes_{\mathcal{B}} 1 \\
& =(-1)^{k(k-1) / 2-n / 2}(-1)^{(n-k)(n-k+1) / 2}(* * \omega) \otimes_{\mathcal{B}} 1 \\
& =(-1)^{k} \omega \otimes_{\mathcal{B}} 1 \\
& =\gamma_{2}(\omega) .
\end{aligned}
$$


Again by (6), (7), (8), (9) we have

$$
c_{\mathrm{L}}(\alpha) \gamma_{21}(\omega)=-\gamma_{21}\left(c_{\mathrm{L}}(\alpha) \omega\right),
$$

so the grading $\gamma_{21}$ on $\mathscr{E}_{21}$ is compatible with the grading on $\mathscr{B}_{\mathrm{gr}}$, and hence $\mathscr{B}_{\mathrm{gr}}=$ End $_{\mathcal{B}}\left(\mathcal{E}_{21}\right)$ as $\mathbb{Z}_{2}$-graded algebras.

Let $\nabla$ be the connection on $\Omega(M)$ induced by the Levi-Civita connection on $T_{\mathbb{R}}^{*} M$. Note that if

$$
\nabla(\omega)=\sum_{i} \nu_{i} \otimes \mu_{i}
$$

for some $\mu_{i} \in \Omega^{k}(M), v_{i} \in \Omega^{1}(M)$, then $d \omega=\sum_{i} v_{i} \wedge \mu_{i}, d^{*} \omega=-\sum_{i} \iota\left(v_{i}\right) \mu_{i}$. Thus,

$$
\left(d+d^{*}\right) \omega=\sum_{i} c_{\mathrm{L}}\left(v_{i}\right) \mu_{i}, \quad\left(d-d^{*}\right) \omega=(-1)^{k} \sum_{i} c_{\mathrm{R}}\left(v_{i}\right) \mu_{i},
$$

namely

$$
d+d^{*}=c_{\mathrm{L}} \circ \nabla, \quad D_{1}=(-1)^{k}\left(d-d^{*}\right)=c_{\mathrm{R}} \circ \nabla .
$$

Define $\hat{\nabla}: \mathcal{E}_{21} \mapsto \mathcal{E}_{21} \otimes_{\mathcal{B}} B\left(\mathscr{H}_{1}\right)$ by

$$
(\hat{\nabla}(\omega))(h)=\sqrt{-1} \sum_{i} \gamma_{1}\left(\mu_{i}\right) \otimes_{\mathcal{B}} c_{\mathrm{R}}\left(v_{i}\right) h
$$

that is,

$$
\hat{\nabla}(\omega)=\sqrt{-1} c_{\mathrm{R}} \circ \nabla\left(\gamma_{1}(\omega)\right)
$$

So $\hat{\nabla}$ is a $\left(\mathscr{A}_{1}, \mathscr{H}_{1}, D_{1}^{\prime}, \gamma_{1}\right)$-connection, and we have

$$
\begin{aligned}
D_{1}^{\prime \varepsilon_{21}}(\omega)=D_{1}^{\prime \varepsilon_{21}}\left(\omega \otimes_{\mathcal{B}} 1\right) & =\left(\gamma_{21} \otimes_{\mathcal{B}} \mathrm{id}\right)(\hat{\nabla}(\omega))(1)+\gamma_{21}(\omega) \otimes_{\mathcal{B}} D_{1}^{\prime}(1) \\
& =\sqrt{-1}\left(\gamma_{21} \otimes_{\mathcal{B}} \mathrm{id}\right) \sum_{i} \gamma_{1}\left(\mu_{i}\right) \otimes_{\mathcal{B}} c_{\mathrm{R}}\left(v_{i}\right) 1+0 \\
& =\sqrt{-1}(-1)^{k} \sum_{i} c_{\mathrm{R}}\left(v_{i}\right) \mu_{i}=\sqrt{-1}\left(d-d^{*}\right) \omega
\end{aligned}
$$

So $D_{2}=D_{1}^{\prime \varepsilon_{21}}$. Therefore we have the Morita equivalence

$$
\begin{aligned}
\left(\mathscr{A}_{2}, \mathscr{H}_{2}, D_{2}, \gamma_{2}\right) & =\left(\operatorname{End}_{\mathscr{A}_{1}}\left(\mathcal{E}_{21}\right), \mathcal{E}_{21} \otimes_{\mathcal{A}_{1}} \mathscr{H}_{1}, D_{1}^{\prime E_{21}}, \gamma_{21} \otimes_{\mathcal{A}_{1}} \gamma_{1}\right) \\
& \sim\left(\mathscr{A}_{1}, \mathscr{H}_{1}, D_{1}, \gamma_{1}\right) .
\end{aligned}
$$

Now let $D_{3}=d+d^{*}$ and $\gamma_{3}=\gamma_{2}=(-1)^{\text {deg }}$. Note that for even-dimensional manifolds,

$$
*^{-1} \sqrt{-1}\left(d-d^{*}\right) *=\left(d+d^{*}\right)(-1)^{\operatorname{deg}+1 / 2}=\sqrt{-1} D_{3} \gamma_{3} .
$$

If we write $\gamma_{3}=\left[\begin{array}{cc}\mathrm{id} & 0 \\ 0 & -\mathrm{id}\end{array}\right]$ and $D_{3}=\left[\begin{array}{cc}0 & T \\ T^{*} & 0\end{array}\right]$, then

$$
*^{-1} D_{2} *=\sqrt{-1} D_{3} \gamma_{3}=\left[\begin{array}{cc}
1 & 0 \\
0 & \sqrt{-1}
\end{array}\right] D_{3}\left[\begin{array}{cc}
1 & 0 \\
0 & -\sqrt{-1}
\end{array}\right] \text {. }
$$


So we have

$$
D_{3}=\left[\begin{array}{cc}
1 & 0 \\
0 & -\sqrt{-1}
\end{array}\right] *^{-1} D_{2} *\left[\begin{array}{cc}
1 & 0 \\
0 & \sqrt{-1}
\end{array}\right] .
$$

Let $C_{\mathrm{gr}}$ be a $\mathbb{Z}_{2}$-graded complex algebra generated by $\Gamma\left(M, T_{\mathbb{R}}^{*} M\right)$ with generator relations as follows:

$$
\left.C_{\mathrm{gr}}:=\left\langle u \in \Gamma\left(M, T_{\mathbb{R}}^{*} M\right)\right| u v+u v=2 g(u, v) \text { for all } u, v \in \Gamma\left(M, T_{\mathbb{R}}^{*} M\right)\right\rangle .
$$

Although $C_{\mathrm{gr}}$ is isomorphic to $B_{\mathrm{gr}}$ through the map

$$
B_{\mathrm{gr}} \rightarrow C_{\mathrm{gr}}: u \mapsto \sqrt{-1} u \text { for all } u \in \Gamma\left(M, T_{\mathbb{R}}^{*} M\right),
$$

we consider a different representation on $\Omega(M)$. Let $c_{\mathrm{L}}^{\prime}$ be the representation of $C_{\mathrm{gr}}$ on $\Omega(M)$ given by

$$
c_{\mathrm{L}}^{\prime}(\sqrt{-1} u)=\left[\begin{array}{cc}
1 & 0 \\
0 & -\sqrt{-1}
\end{array}\right] *^{-1} c_{\mathrm{L}}(u) *\left[\begin{array}{cc}
1 & 0 \\
0 & \sqrt{-1}
\end{array}\right]
$$

for all $u \in \Gamma\left(M, T_{\mathbb{R}}^{*} M\right)$. Then

$$
c_{\mathrm{L}}^{\prime}(u)(\omega)=(-1)^{k-1} *^{-1} c_{\mathrm{L}}(u) * \omega \quad \text { for all } u \in \Gamma\left(M, T_{\mathbb{R}}^{*} M\right) \text { and } \omega \in \Omega^{k}(M) .
$$

Therefore,

$$
c_{\mathrm{L}}^{\prime}(u)=(-1)^{\operatorname{deg}} c_{\mathrm{R}}(u) \quad \text { for all } u \in \Gamma\left(M, T_{\mathbb{R}}^{*} M\right) .
$$

From Kasparov [17], the spectral triple representing Kasparov's fundamental class is supposed to be

$$
\left(\mathcal{A}_{3}, \mathscr{H}_{3}, D_{3}, \gamma_{3}\right)=\left(C_{\mathrm{gr}}, \Omega(M), d+d^{*},(-1)^{\mathrm{deg}}\right),
$$

which is unitarily equivalent to $\left(\mathcal{A}_{2}, \mathscr{H}_{2}, D_{2}, \gamma_{2}\right)$ because of (12) and (13). Thus we have the following theorem.

Theorem 6.2. The projective spectral triple $\left(\mathcal{A}_{1}, \mathscr{H}_{1}, D_{1}, \gamma_{1}\right)$ is Morita equivalent to Kasparov's spectral triple $\left(\mathcal{A}_{3}, \mathscr{H}_{3}, D_{3}, \gamma_{3}\right)$.

For a recent account of Kasparov's fundamental class on noncommutative Riemannian manifolds, we refer the reader to Lord-Rennie-Varilly [19].

\section{Projective spectral triple as fundamental class in $K_{0}\left(M, W_{3}\right)$}

In this section we see how projective spectral triples represent the fundamental classes in the twisted $\mathrm{K}$-homology $K^{0}\left(\mathcal{A}_{W_{3}}\right) \cong K_{0}\left(M, W_{3}\right)$.

Denote by $\boldsymbol{B}_{\text {gr }}$ the $\mathbb{Z}_{2}$-graded algebra of sections of Clifford bundle $\mathrm{Cl}\left(T^{*} M\right)$ over $M$ (even-dimensional only). Then every Clifford module $E$ over $M$ can be considered 
as a finitely generated projective right $\boldsymbol{B}_{\mathrm{gr}}^{\text {op }}$-module, and a Clifford connection $\nabla^{E}$ on $E$ gives rise to a Dirac operator $D^{E}$ on $E$. Then $E \mapsto$ Ind $D^{E}$ defines a canonical homomorphism

$$
K_{0}\left(\boldsymbol{B}_{\mathrm{gr}}^{\text {op }}\right) \stackrel{\text { Ind }}{\longrightarrow} \mathbb{Z} .
$$

By Morita equivalence, $K_{0}\left(\boldsymbol{B}_{\mathrm{gr}}^{\mathrm{op}}\right)$ can be replaced by the K-theory of an ungraded algebra, $K_{0}\left(\mathcal{A}_{W_{3}}\right)$, and the homomorphism Ind becomes the operation of pairing with the projective spectral triple.

Theorem 7.1 (Poincaré duality). For an even-dimensional closed oriented manifold $M$, the projective spectral triple $\varsigma=\left(\mathcal{A}_{W_{3}}, \mathscr{H}_{W_{3}}, D_{W_{3}}, \gamma_{W_{3}}\right)$ represents the twisted $K$-orientation as a cycle of the twisted $K$-homology $K^{0}\left(\overline{\mathrm{A}_{W}}\right) \cong K_{0}\left(M, W_{3}\right)$, and hence gives rise to the Poincaré duality

$$
K^{0}\left(M, W_{3}-c\right) \stackrel{\cap[s]}{\cong} K_{0}(M, c) \quad \text { or } \quad K^{\bullet}(M, c) \times K^{\bullet}\left(M, W_{3}-c\right) \frac{\text { nondegenerate }}{\text { pairing }} \mathbb{Z}
$$

for all $c \in H^{3}(M, \mathbb{Z})$. Here the cap product can be defined by $[\mathcal{E}] \cap[\varsigma]=\left[\varsigma^{\mathcal{E}}\right]$ for any finite Kasparov $C^{\infty}(M)$ - $\mathcal{A}_{W_{3}}$-module $\mathbb{E}$.

For odd-dimensional $M$, the Poincaré duality can be formulated as

$$
K^{\bullet}(M, c) \times K^{\bullet+1}\left(M, W_{3}-c\right) \underset{\text { pairing }}{\stackrel{\text { nondegenerate }}{\longrightarrow}} \mathbb{Z} \quad \text { for all } c \in H^{3}(M, \mathbb{Z}) .
$$

See Kasparov [17], Carey-Wang [5], and Wang [27] for details. When $c$ is 0, this is a special case of the second Poincaré duality theorem [17] in KK-theory.

\section{Local index formula for projective spectral triples}

In this section we present a local index formula associated to the projective spectral triple for every closed oriented Riemannian manifold $M$ of dimension $2 n$. Let $\mathcal{A}=$ $C^{\infty}(M)$. Denote by

$$
\varsigma=(\mathscr{B}, \mathscr{H}, D, \gamma)=\left(\mathcal{A}_{W_{3}}, \mathcal{H}_{W_{3}}, D_{W_{3}}, \gamma_{W_{3}}\right)
$$

the projective spectral triple of $M$ defined in the preceding sections. Suppose that a $\mathrm{K}$-class $[p]$ or $[\mathcal{E}]$ in $K_{0}(\mathscr{B})$ is represented by a projection matrix $p=\left(p_{i j}\right) \in$ $\mathrm{M}_{m}(\mathscr{B})$ or by a right $\mathscr{B}$-module $\mathcal{E}=p \mathscr{B}^{m}$ respectively. Let $D^{\mathscr{E}}$ denote the twisted Dirac operator on $\mathscr{H}^{\mathscr{E}}=\mathscr{E} \otimes_{\mathscr{B}} \mathscr{H}=p \mathscr{H}^{m}$ associated to the projective universal connection $\nabla^{\mathcal{E}}: \mathcal{E} \rightarrow \mathcal{E} \otimes_{\mathcal{B}} \Omega_{u}^{1}(\mathcal{B})$ on $\mathcal{E}$, namely $\nabla^{\mathcal{E}}(p \boldsymbol{b})=p \delta_{u}(p \boldsymbol{b})$ and $D^{\mathscr{E}}(p \boldsymbol{h})=p D(p \boldsymbol{h}), \forall \boldsymbol{b} \in \mathscr{B}^{m}$ for all $\boldsymbol{h} \in \mathscr{H}^{m}$.

The left $\mathscr{B}$-module $\mathscr{H}=\mathscr{H}_{+} \oplus \mathscr{H}_{-}$is $\mathbb{Z}_{2}$-graded and so is $\mathscr{H}^{\mathcal{E}}=\mathscr{H}_{+}^{\mathcal{E}} \oplus \mathscr{H}_{-}^{\mathbb{E}}$. Denote by $D_{ \pm}^{\mathcal{E}}$ the restrictions of $D^{\mathcal{E}}$ to $\mathscr{H}_{ \pm}^{\mathcal{E}} \rightarrow \mathcal{H}_{\mp}^{\mathcal{E}}$. The index of $D^{\mathcal{E}}$ is

$$
\operatorname{Ind}\left(D^{\mathcal{E}}\right)=\operatorname{dim} \operatorname{ker} D_{+}^{\mathcal{E}}-\operatorname{dim} \operatorname{ker} D_{-}^{\mathcal{E}} .
$$


Using the well-known local index formula (cf. [3]), we have

$$
\operatorname{Ind}\left(D^{\mathcal{E}}\right)=\int_{M} \hat{A}(M) \operatorname{ch}\left(\mathscr{H}^{\mathcal{E}} / \mathcal{S}\right) .
$$

$\hat{A}(M)$ is the $\hat{A}$-genus of the manifold $M$,

$$
\hat{A}(M)=\operatorname{det}^{1 / 2}\left(\frac{R / 2}{\sinh (R / 2)}\right) \in \Omega^{\mathrm{ev}}(M) .
$$

The relative Chern character $\operatorname{ch}\left(\mathscr{H}^{\mathcal{E}} / \mathcal{S}\right)$ is explained as follows. We consider $\mathscr{H}$ and $\mathscr{H}^{\mathcal{E}}$ as right Clifford modules with right Clifford actions $c_{\mathrm{R}}$. The connection $\nabla: \mathscr{H} \rightarrow$ $\mathscr{H} \otimes_{\mathcal{A}} \Omega^{1}(M)$ on $\mathscr{H}$ induced by the Levi-Civita connection on $M$ is a right Clifford connection. We can define a right Clifford connection $\nabla^{\mathscr{H}^{\mathcal{E}}}: \mathscr{H}^{\mathcal{E}} \rightarrow \mathscr{H}^{\mathcal{E}} \otimes_{\mathcal{A}} \Omega^{1}(M)$ on $\mathscr{H}^{\mathcal{E}}$ by $\nabla^{\mathscr{H}^{\mathcal{E}}}(p \boldsymbol{h})=p \nabla(p \boldsymbol{h})$. Denote by $R^{\mathscr{H}^{\mathcal{E}}} \in \operatorname{End}_{\mathcal{A}}\left(\mathscr{H}^{\mathcal{E}}\right) \otimes_{\mathscr{A}} \Omega^{2}(M)$ the curvature of the connection $\nabla^{\mathscr{H}^{\mathcal{E}}}$,

$$
R^{\mathscr{H}^{\mathcal{E}}}=\nabla^{\mathscr{H}^{\mathcal{E}}} \nabla^{\mathscr{H}^{\mathcal{E}}}=p(\nabla p)(\nabla p)+p \nabla^{2} \circ p .
$$

Denote by $T$ the twisting curvature, that is, $T=R^{\mathscr{H}^{\mathcal{E}}}-R^{\mathcal{S}}$, where

$$
R^{\mathcal{S}}=c_{\mathrm{R}}(R)=\frac{1}{4} R_{i j k l} c_{\mathrm{R}}\left(e_{l}\right) c_{\mathrm{R}}\left(e_{k}\right) e_{i} \wedge e_{j}
$$

and $R_{i j k l}$ are the components of the Riemannian curvature tensor on $M$ under an orthonormal frame $\left\{e_{i}\right\}$. One can verify that $T=p(\nabla p)(\nabla p)-p c_{\mathrm{L}}(R) p$. With the above notations, the relative Chern character is

$$
\operatorname{ch}\left(\mathscr{H}^{\mathcal{E}} / S\right)=2^{-n} \operatorname{tr} \exp (-T) .
$$

So we have an explicit local index formula

$$
\operatorname{Ind}\left(D^{\mathcal{E}}\right)=2^{-n} \int_{M} \hat{A}(M) \operatorname{tr} \exp \left(-p(\nabla p)(\nabla p)+p c_{\mathrm{L}}(R) p\right) .
$$

From the viewpoint of noncommutative geometry,

$$
\operatorname{Ind}\left(D^{\mathcal{E}}\right)=\langle[p],[\varsigma]\rangle=\langle\operatorname{ch}[p], \operatorname{ch}[\varsigma]\rangle,
$$

where $\operatorname{ch}[p] \in \mathrm{HP}_{0}(\mathscr{B})$ and $\operatorname{ch}[\varsigma] \in \operatorname{HP}^{0}(\mathscr{B})$ are the periodic Connes-Chern characters of $[p]$ and $[\varsigma]$ respectively. On the other hand, in terms of twisted Chern characters, as defined below,

$$
\begin{aligned}
& \operatorname{ch}_{W_{3}}[p]:=\operatorname{Chkr}(\operatorname{ch}[p]) \in H^{\mathrm{ev}}(M, \mathbb{C}), \\
& \operatorname{ch}_{W_{3}}[\varsigma]:=\left(\operatorname{Chkr}^{*}\right)^{-1}(\operatorname{ch}[\varsigma]) \in H_{\mathrm{ev}}(M, \mathbb{C}),
\end{aligned}
$$

the index pairing can be written as

$$
\operatorname{Ind}\left(D^{\mathcal{E}}\right)=\langle[p],[\varsigma]\rangle=\left\langle\operatorname{ch}_{W_{3}}[p], \operatorname{ch}_{W_{3}}[\varsigma]\right\rangle .
$$


We now try to give local expressions of $\operatorname{ch}[p], \operatorname{ch}[\varsigma], \operatorname{ch}_{W_{3}}[p]$, and $\operatorname{ch}_{W_{3}}[\varsigma]$ as well as their relation (15) explicitly. The periodic Connes-Chern character $\operatorname{ch}[p]$ is represented by a sequence of cyclic cycles $\left\{\operatorname{ch}_{0}^{\lambda}(p), \operatorname{ch}_{2}^{\lambda}(p), \ldots\right\}$, where

$$
\operatorname{ch}_{2 m}^{\lambda}(p)=(-1)^{m} \frac{(2 m) !}{m !} \operatorname{tr}\left(p^{\otimes 2 m+1}\right) \in C_{2 m}^{\lambda}(\mathscr{B}) .
$$

This sequence satisfies the periodicity condition $S\left(\operatorname{ch}_{2 m+2}^{\lambda}(p)\right)=\operatorname{ch}_{2 m}^{\lambda}(p)$. An alternative way to represent $\operatorname{ch}[p]$ is to use normalized (b, B)-cycles, that is,

$$
\mathrm{ch}_{2 m}^{(\mathrm{b}, \mathrm{B})}(p)=(-1)^{m} \frac{(2 m) !}{m !} \operatorname{tr}\left(\left(p-\frac{1}{2}\right) \otimes p^{\otimes 2 m}\right) .
$$

As for the Connes-Chern character of $\zeta$, one can apply the Connes-Moscovici [11] local index formula to get a normalized (b, B)-cocycle. However, when trying to derive from Connes-Moscovici's formula an expression in terms of integrals of differential forms on $M$, one will be confronted with a very much involved calculation of Wodzicki residues of various pseudo-differential operators. On the other hand, based on the appearance of formula (14), one can get a $C_{\lambda}$ - $\operatorname{cocycle} \operatorname{ch}_{\lambda}(\varsigma)=\sum_{m} \operatorname{ch}_{\lambda}^{2 m}(\varsigma)$ as follows:

Let $T\left(b_{1}, b_{2}\right)=\left(\nabla b_{1}\right)\left(\nabla b_{2}\right)-b_{1} c_{\mathrm{L}}(R) b_{2}$, and define

$$
\rho_{2 m}^{0}: \mathscr{B}^{\otimes 2 m+1} \rightarrow \Omega^{2 m}(M)
$$

by

$$
\rho_{2 m}^{0}\left(b_{0}, \ldots, b_{2 m}\right)=\frac{1}{(2 m) !} \operatorname{tr}\left(b_{0} T\left(b_{1}, b_{2}\right) \ldots T\left(b_{2 m-1}, b_{2 m}\right)\right) .
$$

Then the relative Chern character $\operatorname{ch}\left(\mathscr{H}^{\mathcal{E}} / \mathcal{S}\right)=2^{-n} \rho_{2 m}^{0}\left(\operatorname{ch}_{2 m}^{\lambda}[p]\right)$.

It is easily seen that $2^{-n} \int_{M} \hat{A}(M) \rho_{2 m}^{0}\left(b_{0}, \ldots, b_{2 m}\right)$ is a Hochschild cocycle, but not a cyclic cocycle if $m \geq 2$. By applying Theorem 2.12, we know that

$$
\rho_{2 m}\left(b_{0}, \ldots, b_{2 m}\right)=\frac{1}{(2 m) !} \operatorname{tr}\left(b_{0} \psi_{2 m}\left(b_{1}, \ldots, b_{2 m}\right)\right)
$$

is a cyclic cocycle and that $\rho_{2 m}\left(\operatorname{ch}_{2 m}^{\lambda}(p)\right)=\rho_{2 m}^{0}\left(\operatorname{ch}_{2 m}^{\lambda}(p)\right)$ for all $p$ with $[p] \epsilon$ $K_{0}(\mathcal{B})$. Thus by Theorem 2.12 and the duality theorem (Theorem 7.1), we have the following conclusions:

Theorem 8.1. The cyclic cocycle $\operatorname{ch}_{\lambda}(\varsigma)=\sum_{m} \operatorname{ch}_{\lambda}^{2 m}(\varsigma)$, where

$$
\operatorname{ch}_{\lambda}^{2 m}(\varsigma)\left(b_{0}, \ldots, b_{2 m}\right)=2^{-n} \int_{M} \hat{A}(M) \rho_{2 m}\left(b_{0}, \ldots, b_{2 m}\right) \quad \text { for all } b_{i} \in \mathcal{B},
$$

represents the Connes-Chern character $\operatorname{ch}[\varsigma]$ of the projective spectral triple $\varsigma$. 
Theorem 8.2. The Connes-Chern character and the twisted Chern character are related by

$$
\operatorname{ch}[\zeta]=\operatorname{ch}_{W_{3}}[\varsigma] \circ \sum_{m} \rho_{2 m} \text { and } \operatorname{ch}_{W_{3}}[p]=\sum_{m} \rho_{2 m}\left(\operatorname{ch}_{2 m}^{\lambda}[p]\right)
$$

as identical periodic cyclic cohomology classes and de Rham cohomology classes respectively.

Corollary 8.3. The twisted Chern characters of $[p]$ and $[\varsigma]$ can be represented by

$$
\operatorname{ch}_{W_{3}}[p]=2^{n} \operatorname{ch}\left(\mathscr{H}^{\mathcal{E}} / \mathcal{S}\right) \text { and } \operatorname{ch}_{W_{3}}[\varsigma]=2^{-n}[\hat{A}(M)] \cap[M]
$$

respectively.

\section{References}

[1] M. Atiyah and G. Segal, Twisted K-theory. Ukr. Math. Bull. 1 (2004), 291-334. Zbl 1151.55301 MR 2172633 http://www.iamm.ac.donetsk.ua/upload/iblock/45e/t1-n3-287-330.pdf

[2] S. Baaj and P. Julg, Théorie bivariante de Kasparov et opérateurs non bornés dans les $C^{*}$-modules hilbertiens. C. R. Acad. Sci. Paris Sér. I Math. 296 (1983), 875-878. Zbl 0551.46041 MR 715325

[3] N. Berline, E. Getzler, and M. Vergne, Heat kernels and Dirac operators. Grundlehren Text Editions, Springer-Verlag, Berlin 2004. Zbl 1037.58015 MR 2273508

[4] P. Bouwknegt, A. L. Carey, V. Mathai, M. K. Murray, and D. Stevenson, Twisted $K$-theory and $K$-theory of bundle gerbes. Comm. Math. Phys. 228 (2002), 17-45. Zbl 1036.19005 MR 1911247

[5] A. L. Carey and B.-L. Wang, Thom isomorphism and push-forward map in twisted $K$ theory. J. K-Theory 1 (2008), 357-393. Zbl 1204.55006 MR 2434190

[6] A. Connes, Non-commutative differential geometry. Inst. Hautes Études Sci. Publ. Math. 62 (1985), 41-144. Zbl 0592.46056 MR 0823176

[7] A. Connes, Noncommutative geometry. Academic Press, San Diego, CA, 1994. Zbl 0818.46076 MR 1303779

[8] A. Connes, On the spectral characterization of manifolds. J. Noncommut. Geom. 7 (2013), 1-82. Zbl 06155603 MR 3032810

[9] A. Connes and A. H. Chamseddine, Inner fluctuations of the spectral action. J. Geom. Phys. 57 (2006), 1-21. Zbl 1105.58004 MR 2265456

[10] A. Connes and M. Marcolli, Noncommutative geometry, quantum fields and motives. Amer. Math. Soc. Colloq. Publ. 55, Amer. Math. Soc., Providence, RI, 2008. Zbl 1209.58007 MR 2371808

[11] A. Connes and H. Moscovici, The local index formula in noncommutative geometry. Geom. Funct. Anal. 5 (1995), 174-243. Zbl 0960.46048 MR 1334867 
[12] J. Cuntz and D. Quillen, Algebra extensions and nonsingularity. J. Amer. Math. Soc. 8 (1995), 251-289. Zbl 0838.19001 MR 1303029

[13] D. S. Freed, Twisted $K$-theory and loop groups. In Proc. Internat. Congr. of Mathematicians, Vol. III, Higher Ed. Press, Beijing 2002 419-430. Zbl 0997.19004 MR 1957550

[14] V. Gayral, J. M. Gracia-Bondía, B. Iochum, T. Schücker, and J. C. Várilly, Moyal planes are spectral triples. Comm. Math. Phys. 246 (2004), 569-623. Zbl 1084.58008 MR 2053945

[15] A. Gorokhovsky, Explicit formulae for characteristic classes in noncommutative geometry. Ph.D. thesis, Ohio State Universtity, Columbus 1999. http://math.colorado.edu/\%7Egorokhov/preprints/sthesis.pdf

[16] A. Jaffe, A. Lesniewski, and K. Osterwalder, Quantum $K$-theory. I. The Chern character. Comm. Math. Phys. 118 (1988), 1-14. Zbl 0656.58048 MR 954672

[17] G. G. Kasparov, Equivariant $K K$-theory and the Novikov conjecture. Invent. Math. 91 (1988), 147-201. Zbl 0647.46053 MR 0918241

[18] J.-L. Loday, Cyclic homology. 2nd ed., Grundlehren Math. Wiss. 301, Springer-Verlag, Berlin 1998. Zbl 0885.18007 MR 1600246

[19] S. Lord, A. Rennie, and J. C. Várilly, Riemannian manifolds in noncommutative geometry. J. Geom. Phys. 62 (2012), 1611-1638. Zbl 1261.53032 MR 2922025

[20] V. Mathai, R. B. Melrose, and I. M. Singer, Fractional analytic index. J. Differential Geom. 74 (2006), 265-292. Zbl 1115.58021 MR 2258800

[21] V. Mathai and D. Stevenson, On a generalised Connes-Hochschild-Kostant-Rosenberg theorem. Adv. Math. 200 (2006), 303-335. Zbl 1098.19002 MR 2200848

[22] B. Mesland, Bivariant K-theory of groupoids and the noncommutative geometry of limit sets. Bonner Math. Schriften 394, Rheinische Friedrich-Wilhelms-Universität, Bonn 2009. Zbl 06231907

[23] D. Quillen, Superconnections and the Chern character. Topology 24 (1985), 89-95. Zbl 0569.58030 MR 790678

[24] I. Raeburn and D. P. Williams, Morita equivalence and continuous-trace $C^{*}$-algebras. Math. Surveys Monographs 60, Amer. Math. Soc., Providence, RI, 1998. Zbl 0922.46050 MR 1634408

[25] M. A. Rieffel, Morita equivalence for $C^{*}$-algebras and $W^{*}$-algebras. J. Pure Appl. Algebra 5 (1974), 51-96. Zbl 0295.46099 MR 0367670

[26] J. Rosenberg, Continuous-trace algebras from the bundle theoretic point of view. J. Austral. Math. Soc. Ser. A 47 (1989), 368-381. Zbl 0695.46031 MR 1018964

[27] B.-L. Wang, Geometric cycles, index theory and twisted $K$-homology. J. Noncommut. Geom. 2 (2008), 497-552. Zbl 1169.19003 MR 2438341

Received September 22, 2010; revised February 17, 2012

D. Zhang, Mathematics 253-37, California Institute of Technology, Pasadena, CA 91125, U.S.A.

E-mail: dapeng.zhang@gmail.com 